\title{
Glacier Surge Mechanism Based on Linked Cavity Configuration of the Basal Water Conduit System
}

\author{
BARCLAY KAMB \\ Division of Geological and Planetary Sciences, California Institute of Technology, Pasadena
}

\begin{abstract}
Based on observations of the 1982-1983 surge of Variegated Glacier, Alaska, a model of the surge mechanism is developed in terms of a transition from the normal tunnel configuration of the basal water conduit system to a linked cavity configuration that tends to restrict the flow of water, resulting in increased basal water pressures that cause rapid basal sliding. The linked cavity system consists of basal cavities formed by ice-bedrock separation (cavitation), $\sim 1 \mathrm{~m}$ high and $\sim 10 \mathrm{~m}$ in horizontal dimensions, widely scattered over the glacier bed, and hydraulically linked by narrow connections where separation is minimal (separation gap $\leq 0.1 \mathrm{~m}$ ). The narrow connections, called orifices, control the water flow through the conduit system; by throttling the flow through the large cavities, the orifices keep the water flux transmitted by the basal water system at normal levels even though the total cavity cross-sectional area $\left(\sim 200 \mathrm{~m}^{2}\right)$ is much larger than that of a tunnel system $\left(\sim 10 \mathrm{~m}^{2}\right)$. A physical model of the linked cavity system is formulated in terms of the dimensions of the "typical" cavity and orifice and the numbers of these across the glacier width. The model concentrates on the detailed configuration of the typical orifice and its response to basal water pressure and basal sliding, which determines the water flux carried by the system under given conditions. Configurations are worked out for two idealized orifice types, step orifices that form in the lee of downglacier-facing bedrock steps, and wave orifices that form on the lee slopes of quasisinusoidal bedrock waves and are similar to transverse "N channels." The orifice configurations are obtained from the results of solutions of the basal-sliding-with-separation problem for an ice mass constituting a near half-space of linear rheology, with nonlinearity introduced by making the viscosity stress-dependent on an intuitive basis. Modification of the orifice shapes by melting of the ice roof due to viscous heat dissipation in the flow of water through the orifices is treated in detail under the assumption of local heat transfer, which guarantees that the heating effects are not underestimated. This treatment brings to light a meltingstability parameter $\Xi$ that provides a measure of the influence of viscous heating on orifice cavitation, similar but distinct for step and wave orifices. Orifice shapes and the amounts of roof meltback are determined by $\Xi$. When $\Xi \geq 1$, so that the system is "viscous-heating-dominated," the orifices are unstable against rapid growth in response to a modest increase in water pressure or in orifice size over their steady state values. This growth instability is somewhat similar to the jökulhlaup-type instability of tunnels, which are likewise heating-dominated. Whien $\Xi \leq 1$, the orifices are stable against perturbations of modest to even large size. Stabilization is promoted by high sliding velocity $v$, expressed in terms of a $v-1 / 2$ and $v-1$ dependence of $\Xi$ for step and wave cavities. The relationships between basal water pressure and water flux transmitted by linked cavity models of step and wave orifice týpe are calculated for an empirical relation between water pressure and sliding velocity and for a particular, reasonable choice of system parameters. In all cases the flux is an increasing function of the water pressure, in contrast to the inverse flux-versuspressure relation for tunnels. In consequence, a linked cavity system can exist stably as a system of many interconnected conduits distributed across the glacier bed, in contrast to a tunnel system, which must condense to one or at most a few main tunnels. The linked cavity model gives basal water pressures much higher than the tunnel model at water fluxes $\geq 1 \mathrm{~m}^{3 / s}$ if the bed roughness features that generate the orifices have step heights or wave amplitudes less than about $0.1 \mathrm{~m}$. The calculated basal water pressure of the particular linked cavity models evaluated is about 2 to 5 bars below ice overburden pressure for water fluxes in the range from about 2 to $20 \mathrm{~m}^{3 / \mathrm{s}}$, which matches reasonably the observed conditions in Variegated Glacier in surge; in contrast, the calculated water pressure for a single-tunnel model is about 14 to 17 bars below overburden over the same flux range. The contrast in water pressures for the two types of basal conduit system furnishes the basis for a surge mechanism involving transition from a tunnel system at low pressure to a linked cavity system at high pressure. The parameter $\Xi$ is about 0.2 for the linked cavity models evaluated, meaning that they are stable but that a modest change in system parameters could produce instability. Unstable orifice growth results in the generation of tunnel segments,
\end{abstract}

Copyright 1987 by the American Geophysical Union.

Paper number 6B6328.

$0148-0227 / 87 / 006 \mathrm{~B}-632 \$ 05.00$ 
which may connect up in a cooperative fashion, leading to conversion of the linked cavity system to a tunnel system, with large decrease in water pressure and sliding velocity. This is what probably happens in surge termination. Glaciers for which $\Xi \leq 1$ can go into surge, while those for which $\Xi \geq 1$ cannot. Because $\Xi$ varies as $\alpha^{3 / 2}$ (where $\alpha$ is surface slope), low values of $\Xi$ are more probable for glaciers of low slope, and because slope correlates inversely with glacier length in general, the model predicts a direct correlation between glacier length and probability of surging; such a correlation is observed (Clarke et al., 1986). Because $\Xi$ varies inversely with the basal shear stress $\tau$, the increase of $\tau$ that takes place in the reservoir area in the buildup between surges causes a decrease in $\Xi$ there, which, by reducing $\Xi$ below the critical value $\sim 1$, can allow surge initiation and the start of a new surge cycle. Transition to a linked cavity system without tunnels should occur spontaneously at low enough water flux, in agreement with observed surge initiation in winter.

\section{INTRODUCTION}

The fastest known glacier flow motions occur in glacier surges. There has been much theorizing as to the cause(s) of the fast flow (summarized by Paterson [1981, p. 288]), but few if any firm conclusions have emerged because observations of the processes in action, which could guide physical reasoning, have been inadequate. Studies of the 1982-1983 surge of Variegated Glacier, Alaska, provide a new, enlarged body of observations [Kamb et al., 1985]. I present here a physical model of the surge mechanism developed on the basis of these observations. A brief sketch of the model has been given by Kamb et al. [1985, p. 478]. While the treatment is based on a "hard bed" model of the surge mechanism, it seems likely, as explained in section 10, that a number of the important results are also applicable at least qualitatively to "soft bed" models, in which sliding is over deformable basal till. The discussion concentrates on the mechanism of surging in spring and summer when relatively large amounts of water are available to the basal water conduit system. The surge mechanism in wintertime can be considered by extending the concepts developed here to conditions of low water flow; this will be done in a subsequent paper.

\section{OBSERVATIONAL BASIS}

The following observations from the surge of Variegated Glacier [Kamb et al., 1985; Raymond, this issue] form the direct basis of the surge model:

1. The fast flow motion during the surge is due to rapid basal sliding.

2. During surge, the pressure of water in the basal conduit system is high, within $2-5$ bars of the ice overburden pressure, and occasionally reaching overburden; in the nonsurging state it is distinctly lower, generally 4-16 bars below overburden, but with occasional peaks to higher levels. Peaks in pressure, particularly those in which the water pressure rises to near overburden, correspond to peaks in sliding motion, both in surge and out. These facts are taken as indication that the direct cause of the high sliding speed in surge is high basal water pressure.

3. Major slowdowns in surge motion, and particularly surge termination, are accompanied by large flood peaks in the terminus outflow streams and by a drop of the glacier surface by $0.1-0.7 \mathrm{~m}$. This, in conjunction with observations of uplift followed by drop of the glacier surface in minisurges [Kamb and Engelhardt, 1987], is interpreted as an indication that the high sliding speeds and high basal water pressures in surge and in minisurges are coupled with extensive basal cavitation, as expected theoretically [Lliboutry, 1968; Kamb, 1970, p. 720; Iken, 1981; Fowler, 1987].

4. The flow of water through the basal water conduit system, as indicated by dye-tracing experiments, is much slower in surge than in the nonsurging state [Brugman, 1986]. After surge termination the mean water transport speed along the length of the glacier (lower half) was $0.7 \mathrm{~m} / \mathrm{s}$, typical of basal water flow speeds found in nonsurge-type glaciers; during surge, in contrast, the mean water transport speed was only $0.025 \mathrm{~m} / \mathrm{s}$.

5. In the dye-tracing experiment during surge the dye was dispersed across the width of the glacier, appearing in all outflow streams, whereas after surge termination the dye appeared in one stream only.

6. The outflow stream water during the surge was extremely turbid (suspended sediment content at concentration $\sim 100 \mathrm{~kg} / \mathrm{m}^{3}$ for particle sizes $\leq 10 \mu \mathrm{m}$ ), much more turbid than after surge or in normal, nonsurging glaciers (sediment content $\sim 1-10 \mathrm{~kg} / \mathrm{m}^{3}$ ) [Brugman, 1986, p. 88].

\section{FORMULATION OF A MODEL OF THE SURGE MECHANISM}

The foregoing observations throw a sharp focus on what needs to be explained by a physical model of the surge mechanism. The high sliding speeds are explained by the high basal water pressures. A detailed explanation requires a detailed model of the relation between basal water pressure and sliding speed. Such a model can be developed within the framework of the basal sliding mechanism discussed here (or see Fowler [1986]), but because, as will be argued, the detailed relationship between water pressure and sliding speed is not the essential element of the model needed to explain surging, I will here pass over these details and instead assume a simple empirical relation between water pressure and sliding.

The essential ingredient of the surge model is what causes the high basal water pressures in surge. How is the high basal water pressure maintained and indeed enhanced in spring and early summer, when, according to the standard model of the basal water conduit system [Röthlisberger, 1972], the pressure should drop as an increasing flux of water is carried by the system? Since high basal water pressure and high basal sliding promote basal cavitation, opening up holes (cavities) through which water could move at the base of the glacier, and thus increasing the hydraulic conductivity of the basal water conduit system, why does the water not drain out from under the glacier more rapidly than in nonsurge and thereby reduce the water pressure to subnormal values? Why, on the contrary, does the glacier in surge show an unusually high "retentivity" for water, as shown by the abnormally low water transport speed revealed by dye tracing? These questions go to the heart of what is in my view the essential physical difference between the surging and nonsurging states of the glacier. The surge model concentrates on explaining this difference and is therefore in the first instance a model of the basal water conduit system in surge.

The conduit system that dominates the transport of water in 
the nonsurging state is a basal tunnel system of the kind discussed in theoretical terms by Röthlisberger [1972], Weertman [1972], Nye [1976], Spring and Hutter [1981, 1982], and Lliboutry [1983]. It consists of one or two main tunnels, of order 1 or a few meters in diameter, running along the length of the glacier at the bed, usually near the center or deepest part, and probably fed by smaller side tunnels heading in glacier moulins.

The water conduit system in the surging state is very different. This is shown by the dye tracer experiments in terms of the slow transport speed of water through the system and the high dispersion of the injected dye pulse [Brugman, 1986]. The system cannot consist of the normal tunnels of the nonsurging state with addition of conduits formed by basal cavitation under the high basal water pressure and rapid basal sliding because in this case the water transport speed would remain high and the total water transport (flux) would be increased so that, as noted above, the high water pressure could not be maintained, at least without an abnormally large throughput of water, which is not observed. It follows that the normal tunnel system must not be present.

In order to transport about $5 \mathrm{~m}^{3 / \mathrm{s}}$ of water at an average longitudinal speed of $0.025 \mathrm{~m} / \mathrm{s}$, the conduit system must have a total cross-sectional area of about $200 \mathrm{~m}^{2}$ in the transverse plane of the glacier. (An estimated $5 \mathrm{~m}^{3 / \mathrm{s}}$ is the average flux at the time of the tracer experiment, taking into account the distributed input of meltwater from the tracer injection point to the terminus, where the discharge was $7 \mathrm{~m}^{3 / \mathrm{s}}$.) If the conduits are basal cavities, their average height, areally averaged across the $1-\mathrm{km}$ width of the glacier including areas of ice-bed contact (where the height is 0 ), is $0.2 \mathrm{~m}$. This is compatible with the average height $0.1 \mathrm{~m}$ inferred from the drop in elevation of the ice surface on surge termination and also with the excess amounts of water released from the glacier at surge termination and during the 4 days thereafter, which correspond to a drop in average cavity height of 0.1 and $0.3 \mathrm{~m}$, respectively. (These are upper limits because some of the surface drop may have been due to ice strain and some of the released water may have been stored in intraglacial porosity; see Kamb et al. [1985, p. 477].) Basal cavities of height $\sim 1-2 \mathrm{~m}$, distributed widely over the glacier bed and occupying $\sim 10-20 \%$ of the bed area, would provide passageways for water flow of the dimensions needed and would involve the widespread contact between basal water and the ice-bed interface that could account for the extremely high turbidity of the o, $\mu$ tflow water in surge (section 2, observation 6). The pattern of basal cavitation visualized seems reasonable in relation to cavities that have been actually observed under glaciers [Carol, 1947; Kamb and La Chapelle, 1964; Vivian and Bocquet, 1973] or that can be inferred from detailed observations of the abrasion markings on former glacier beds.

If the water conduit system in surge consisted of an openly interconnected network of basal cavities of the dimensions suggested, the water transport speed through it would be $\sim 1 \mathrm{~m} / \mathrm{s}$, as it is through normal tunnel systems, the lateral dimensions of tunnels being of the same order. This is in strong contradiction with the observed transport speed, $0.025 \mathrm{~m} / \mathrm{s}$. It follows that the water flow through the conduits of $200 \mathrm{~m}^{2}$. cross-sectional area must be throttled in some way. There is a natural reason why this should happen. In the sliding of ice over an irregularly undulatory bed, the distribution of normal stress across the ice-bedrock interface, which controls ice-bed separation, is such that the large cavities that form tend to be isolated from one another, so that the water in them tends not to communicate hydraulically. (A long steplike or wavelike roughness feature running transverse to the ice flow would develop a long, connected cavity, but the transverse direction of hydraulic communication in it would not aid longitudinal transport of water.) When the basal water pressure becomes high enough and the sliding velocity rapid enough, cavitation in the bed areas intervening between the large cavities develops sufficiently to provide hydraulic connections between the cavities, but the connections are small features, much smaller than the cavities they connect. It is this system of hydraulically linked cavities that I here consider in a model of the surge mechanism. From the evidence previously discussed, it appears that most of the pressure drop or potential drop in the water flow through the linked cavity system occurs in the narrow connections, or orifices, as I will call them, and as a consequence, these orifices throttle and control the flow. On the other hand, for a parcel of water traveling through the system, most of its time is spent moving slowly through the large cavities, so that the overall transit time is tied to the $200 \mathrm{~m}^{2}$ total cross-sectional area of the cavities. The water flow through the orifices is probably fast and turbulent, as will be seen later, and the water from each orifice probably emerges as a jet into the cavity downstream, which helps to explain how the water is able to pick up and carry a large amount of fine sediment in suspension.

Once the basic topology of the water conduit system in surge is ascertained, it becomes possible to formulate a physical model of it in sufficient detail to permit its hydraulic properties to be determined, analogously to what has been done for the normal tunnel system by Röthlisberger [1972]. The results provide a basis for deciding whether the underlying picture of the surge mechanism is satisfactory and for identifying the physical conditions that distinguish the surging and nonsurging states of glacier motion, from which one can reason about what causes a glacier to be in the nonsurging or surging state.

\section{THE LINKED CAVITY MODEL}

The pattern of basal cavitation visualized in the linked cavity model is shown schematically in Figures 1 and 2. Figure 2 shows schematically the longitudinal cross-sectional shapes of the leeside ice-bed separation cavities whose plan view shapes are shown in Figure 1. The hachured lines in Figure 1 represent upstream cavity boundaries where the ice separates from bedrock, often at the edge of a topographic step or sharp break in slope of the bedrock surface, as shown in Figure 2; the nonhachured lines in Figure 1 are where the ice recontacts the bed downstream. The cavitation pattern in Figure 1 is rather similar to natural examples of basal cavitation mapped by Walder and Hallet [1979, Figure 7] and by Hallet and Anderson [1980, p. 174], except for the presence of solution-etched flow channels in the limestone bedrock of these natural examples. Under high sliding velocity, the cavities probably elongate greatly downglacier, but the basic topology of the hydraulic linkage of cavities remains similar.

In Figure 1, the larger areas of the ice-bedrock separation represent the cavities of the linked cavity model, and the narrow connections between these are the separation-gap orifices. In cross section these features appear as shown in Figure 2: Figure $2 a$ is a section through two separation cavities in succession along the flow line, while Figure $2 b$ is a section through two orifices, somewhat exaggerated in size for clarity.

The pattern of water flow through the linked cavity system is shown by the small arrows in Figure 1 . Because of the geometry of cavitation, the flow water tends to be in the lateral direction, particularly in the orifices. This lateral flow is reflected in the 


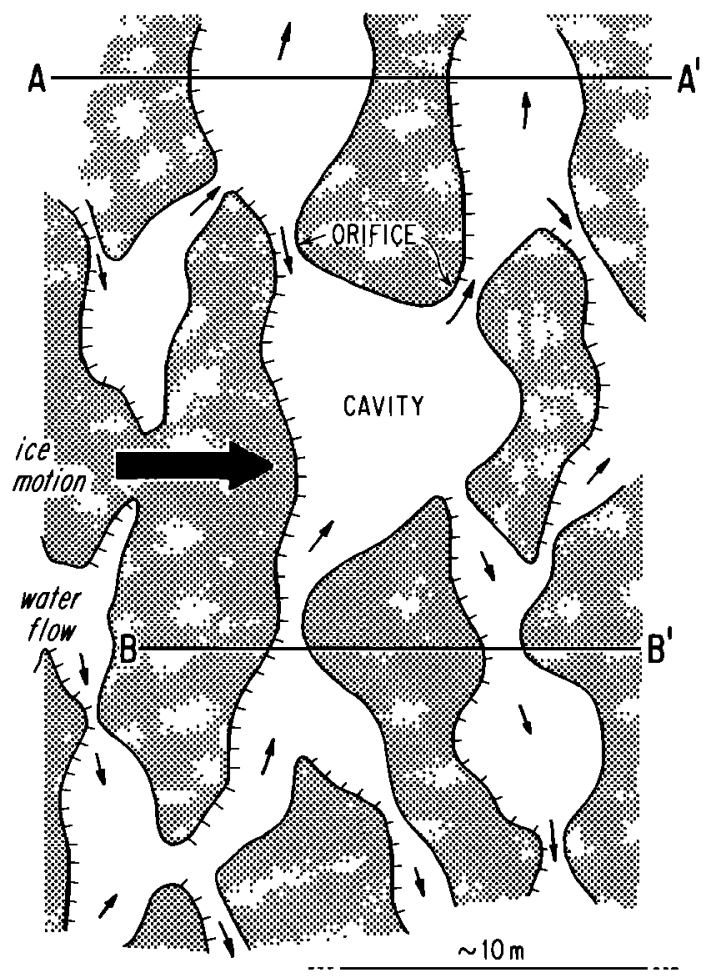

Fig. 1. Conception of the linked cavity basal water conduit system, in map view, portraying schematically a small area of the glacier bed, of lateral dimensions $\sim 20 \mathrm{~m}$. Areas of ice contact with the bed are shaded, areas of ice-bed separation (cavitation) are blank. Vertical cross sections along lines $\mathrm{AA}^{\prime}$ and $\mathrm{BB}^{\prime}$ are shown in Figure 2. The large arrow indicates the direction of basal sliding. Ice separates from the bed along the hachured lines and recontacts the bed along the plain lines. One large cavity and two small "onfices" in the linked cavity pattern are identified. Directions of water flow through the system are shown with small arrows. Approximate scale is indicated by the $10-\mathrm{m}$ bar.

wide lateral dispersion of dye in the tracer experiment during surge (section 2, observation 5).

In developing a quantitative model of the linked cavity system I will consider the system in terms of a typical cavity and a typical linking orifice, illustrated in plan view in Figure 3. The dimensions associated with the cavity and orifice, marked in Figure 3, are as follows:

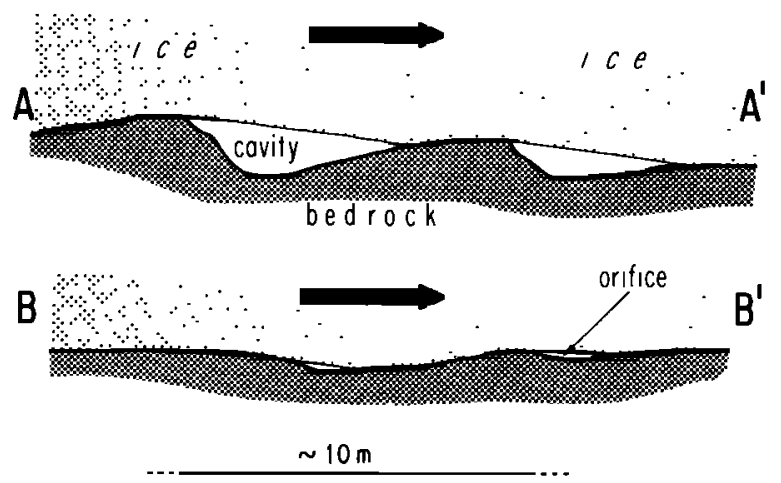

Fig. 2. Vertical cross sections through the schematic linked cavity system of Figure 1, along lines $\mathrm{AA}^{\prime}$ and $\mathrm{BB}^{\prime}$. Heavy shading indicates bedrock, light shading ice. The arrows show the direction of basal sliding. The blank areas are water-filled volumes formed by ice-bed separation (cavitation) in the sliding process. Section $\mathrm{AA}^{\prime}$ shows two large separation cavities, while $\mathrm{BB}^{\prime}$ shows two separation gap orifices, whose gap height is exaggerated for visibility in the drawing. Approximate scale is indicated by the $10-\mathrm{m}$ bar.

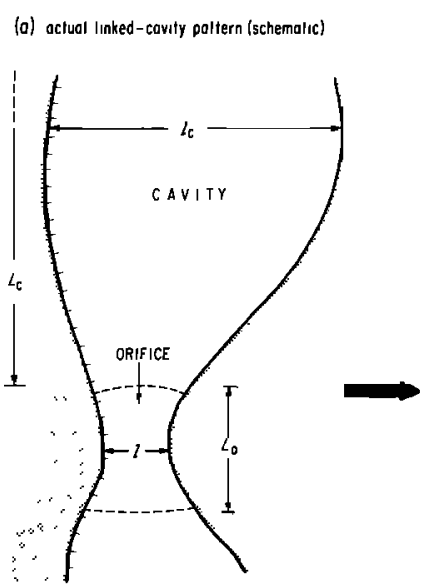

(b) idealized linhed-covily model

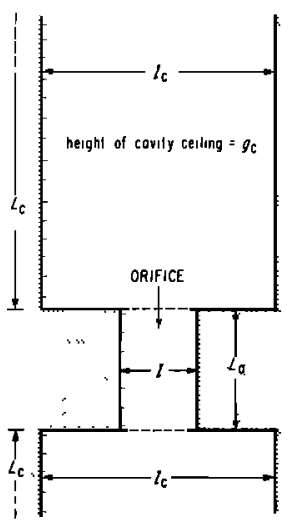

Fig. 3. Local detail and dimensions in the linked cavity system as seen in map view, with conventions of Figure 1. (a) A schematic representation of a realistic linkage pattern between two cavities. (b) The idealized geometry of the linkage assumed in the linked cavity model. The length, breadth, and height parameters $L, l$, and $g$ are discussed in the text.

$l$ is the average lateral dimension (breadth) of the orifice in the direction transverse to water flow. This is always in the direction of basal sliding and represents the length of the separation gap between ice and bedrock, which forms the orifice.

$l_{c}$ is the average dimension (breadth) of the cavity in the direction transverse to water flow. In the cavitation pattern of Figure 1 this dimension is generally parallel to basal sliding, but in downglacier-elongated cavities it tends to be transverse to sliding.

$L_{\mathrm{o}}$ is the length of the orifice in the direction parallel to water flow. Because of the curving, sinuous pattern of the ice-bedrock separation lines and recontacting lines (Figure 1), the dimension $L_{\mathrm{o}}$ is not sharply defined, as Figure $3 a$ suggests, but in the model the distinction between cavity and orifice is sharpened by idealizing their geometry in the way shown in Figure $3 b$.

$L_{\mathrm{c}}$ is the dimension of the cavity in the direction parallel to water flow, or, more precisely, the distance between successive orifices along the water-flow path.

$\Lambda=L_{\mathrm{c}} / L_{\mathrm{o}}$ is the "head gradient concentration factor" (section 5).

$g_{\mathrm{c}}$ is the average height of the cavity.

$g$ is the local height (measured perpendicular to the bedrock surface) of the separation-gap orifice. It is a function of position across the orifice, from the point of ice-bedrock separation to the point of recontact.

$N_{\mathrm{o}}$ is the number of independent orifices in a transverse section across the glacier. The average lateral spacing between independent orifices is $W / N_{\mathrm{o}}$, where $W$ is the glacier width. By independent orifices I mean orifices that are not in succession along water flow paths, so that their water fluxes add to make the total water flux carried by the system.

An actual linked cavity system is an ensemble of cavities and orifices of different shapes and sizes, resulting from cavitation by basal sliding over the diverse and sundry roughness features of the bed, under a spatial distribution of basal shear stress, water pressure, and ice overburden pressure. The complexity of the actual system is suppressed in the model developed here by replacing the spectrum of cavity and orifice dimensions with a single set of "typical" dimensions as indicated above and by considering how these dimensions are controlled by a single set of values of basal shear stress $\tau$, water pressure $P_{\mathrm{W}}$, and ice overburden pressure $P_{\mathrm{l}}$, when basal sliding takes place over 
roughness features of a single type, with prescribed dimensions. This idealization, which is as great a simplification of the system as can be made without losing what I regard as its essential physical characteristics, is chosen here as the first approximation to the behavior of the complicated natural system. It is made in the same spirit as the analysis of basal sliding over a bed with a single type of roughness feature [e.g., Weertman, 1957]).

Because the flow of water through the linked cavity system is controlled by the orifices, as discussed in section 3, and because the orifices are the ice-bed separation gaps that are most sensitive to the conditions controlling separation, the hydraulic behavior of the system is much more sensitive to the detailed geometry of the orifices than it is to the cavities. The cavities doubtless vary somewhat as physical conditions change, but the variation of the orifices has a much larger effect on the behavior of the system. Consequently, in analyzing the linked cavity model I will simply assign reasonable values to $l_{c}, L_{c}$, and $g_{c}$ and will concentrate the effort on how the orifice dimensions $l$ and $g$ are determined by the sliding process and by water pressure and flow. This is another simplification that can reasonably be made in a first treatment of the system.

There are three distinct physical processes that, acting together, determine the hydraulic behavior of the linked cavity model: (1) for given roughness characteristics of the bed, given $P_{\mathrm{W}}$ and $P_{\mathrm{I}}$ and given sliding velocity $\nu$, there is a particular orifice geometry determined by the cavitation process in basal sliding; (2) for given orifice and cavity geometry and given hydraulic gradient, there will be a certain flow of water through the linked cavity system; and (3) the water flow will result in generation of heat by viscous dissipation, which will cause enlargement of the orifices by melting of the ice roof, resulting in a modification of the results of process 1 and a consequent increase in the flow given by process 2 . Since the effect of heat dissipation is crucial in the functioning of a tunnel system and since for a given total water flux down a given hydraulic gradient the dissipation of heat will be the same in a tunnel system and a linked cavity system, it is essential to take it into account in the linked cavity model.

Although there is a considerable parallelism between the treatment of the linked cavity model here and the treatment of the tunnel model by Röthlisberger [1972], the two models differ substantially because the water flow and ice flow geometries are very different and because the tunnel model lacks a component process of type 1 above. The linked cavity conduits have an essential feature of the " $\mathrm{N}$ channels" introduced by Nye [1973] and considered by Weertman [1972, p. 306], namely, that they are tied to specific topographic features of the bed. They differ in this respect fundamentally from tunnels ("R channels" of Weertman [1972]), which, in the model concept of Röthlisberger [1972], are not tied to any topographic features of the bed. The "N channels" were specifically assumed to follow bedrock channels incised by erosion into the bed, whereas the linked cavity conduits considered here are tied to bedrock roughness features of diverse kinds.

\section{WATER FLOW AND VISCOUS HEATING}

Given the geometry of the linked cavity model as specified in section 4 , the flow of water through the system can be calculated as follows. Call $\alpha_{w}$ the overall longitudinal hydraulic gradient (slope of the hydraulic grade line) along the length of the glacier. In the evaluation here, $\alpha_{w}$ is taken to be equal to $\alpha$, the surface slope of the glacier, but this is not a necessary assumption. If the linked cavity system has an average tortuosity $\omega$, the average hydraulic gradient along the water flow paths is then $\alpha / \omega$. The model condition that the orifices control or throttle the flow is equivalent to the statement that all drop in hydraulic head is taken across the orifices; hence the head gradient in the orifices is the average gradient multiplied by $L_{\mathrm{c}} / L_{\mathrm{o}}=\Lambda$ (section 4 ). The orifice gaps are assumed to be thin compared to their breadth $(g \ll l)$, as seems appropriate for narrow ice-bedrock separation gaps that are only marginally open. For simplicity, the typical orifice is taken to have a cross-sectional shape that is constant along its length $L_{0}$. The flow of water through the orifice is then determined locally by the local gap height $g(x)$, which depends on a spatial coordinate $x$ across the breadth $l$ of the orifice. Since the flow is turbulent (as will be shown), it can be obtained from the Manning formula [Röthlisberger, 1972, equation (9)] by putting the hydraulic radius equal to $g(x) / 2$ :

$$
\bar{u}_{\mathrm{W}}(x)=M^{-1}\left(\frac{g}{2}\right)^{\frac{2}{3}}\left(\frac{\alpha \Lambda}{\omega}\right)^{\frac{1}{2}}
$$

Here $\bar{u}_{W}$ is the mean water flow velocity (averaged across the gap height $g), M$ is the Manning roughness, and $\alpha \Lambda / \omega$ is the local hydraulic gradient in the orifice as discussed above. The total flux of water $Q_{\mathrm{W}}$ carried by the linked cavity system is obtained by multiplying (1) by the local gap width $g(x)$, integrating over the breadth $l$ of the orifice, and summing the contributions from the $N_{0}$ independent orifices (section 4):

$$
Q_{\mathrm{w}}=\frac{N_{\mathrm{o}}}{2^{2 / 3} M}\left(\frac{\alpha \Lambda}{\omega}\right)^{\frac{1}{2}} \int_{0}^{l}[g(x)]^{\frac{5}{3}} d x
$$

The local rate of heat generation by viscous heating is the local water flux $\bar{u}_{\mathrm{w} g} \mathrm{times}$ the potential gradient $\rho_{\mathrm{w}} g_{\mathrm{r}} \alpha \Lambda / \omega$ (where $g_{\mathrm{r}}$ is gravity and $\rho_{\mathrm{W}}$ is the density of water). Expressed in terms of an equivalent volume rate of melting of ice by dividing by $\rho_{I} H$, where $H$ is the latent heat of melting and $\rho_{I}$ is the ice density, the local heat generation rate, per unit area of the orifice roof, is thus

$$
\dot{m}=\frac{(\alpha \Lambda / \omega)^{3 / 2}}{2^{2 / 3} D M} g^{5 / 3}
$$

where $D=\rho_{\mathrm{I}} H / \rho_{\mathrm{w}} g_{\mathrm{r}}$ is a constant with dimensions of length $(D=$ $31 \mathrm{~km})$.

The rate of melting caused by the heat generation in (3) is governed by heat transfer from the water to the ice roofs of the orifices and cavities. A fully detailed treatment based on the principles of heat transfer, analogous to that carried out for tunnel systems by Spring and Hutter [1981, 1982], is rather complicated and will here be avoided by the simple assumption that the heat generated is transferred locally to the ice roof, as was assumed in the original treatments of tunnel systems [Röthlisberger, 1972; Nye, 1976]. This assumption guarantees that the effects of heat generation on the linked cavity model will not be underestimated, because a heat transfer distributed over the cavity roofs as well as the orifices would reduce the melting in the orifices and thus reduce its effect on the hydraulic behavior of the linked cavity system. Under this assumption and neglecting the effect of the pressure dependence of the melting point [Röthlisberger, 1972, p. 179], which averages to zero if $\alpha_{w}=\alpha$, equation (3) gives the meltback rate of the orifice roof.

\section{BASAL CAVITATION}

The sizes and shapes of basal cavities and separation gap orifices of the linked cavity model (Figure 2) are determined by 
(a)
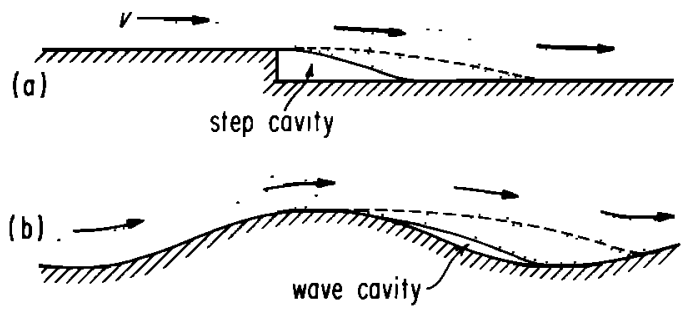

(c)

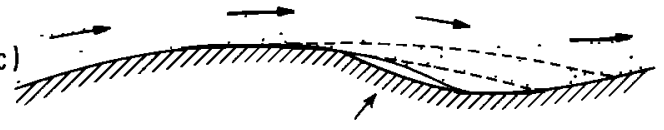

hybrid-type covities

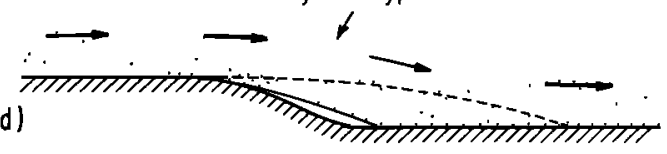

(e)

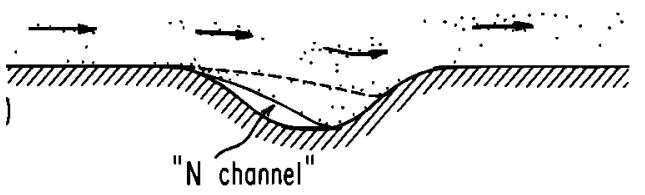

Fig. 4. Idealized geometry (schematic) of ice-bed separation gaps formed by cavitation in basal sliding, seen in vertical cross section parallel to the sliding direction (arrows). Ice is stippled, bedrock cross-hatched; separation gaps are blank. The dashed lines show the configuration of the cavity roofs at increased sliding velocity and/or basal water pressure. (a) A step cavity (or step orifice) formed in the lee of a downglacierfacing bedrock step. (b) A wave cavity on the lee slope of a bedrock wave. (c) Cavitation develops in the lee of a faceted wave such as is often seen on glaciated bedrock surfaces; it has characteristics that are a hybrid of Figures $4 a$ and $4 b$. (d) A different type of hybrid cavity, developed in the lee of a rounded bedrock step. (e) Cavitation in a transverse "N channel" of the kind visualized by Weertman [1972]. The scale of these features is arbitrary, but for the separation gap orifices in the linked cavity system associated with surging as considered here the step or wave heights are $\sim 0.1 \mathrm{~m}$.

the mechanics of ice-bedrock separation. Before the effects of heat dissipation by water flow through the linked cavity system are brought into consideration, separation can be treated as a purely mechanical consequence of the sliding of ice over bedrock topography under the action of basal water pressure [Lliboutry, $1968 ; K a m b, 1970$, section $19 ;$ Fowler, 1986]. If at any point on the ice-bedrock interface the normal pressure across the interface drops below the basal water pressure, then an ice-bed separation cavity will start to open at this point, provided that water under the given pressure can actually gain access to the cavity. The extent and shape of the cavity that develops is in principle determined by the solution of a mixed-boundary-value problem in ice flow mechanics, the boundary condition being a constraint on the stress over the separated parts of the ice sole and a constraint on the ice flow velocity over the nonseparated parts. The problem is tricky because the areas of basal separation, which are the areas where the stress boundary condition is to be applied, are not prescribed in advance but must be determined as part of the solution. From the solution one obtains the breadth dimension $l$ for the orifices of the model, and the distribution of gap height $g(x)$ over the breadth of the orifices. The dimensions $l_{\mathrm{c}}$ and $g_{\mathrm{c}}$ for the cavities are determined physically in the same way, but the model is not concerned in detail with them, as explained in section 4 .

Two types of bedrock topographic features, illustrated in idealized form in Figures $4 a$ and $4 b$, have very different basal separation characteristics and need to be distinguished in the linked cavity model. The first (Figure $4 a$ ) is a downglacier-facing step in the bedrock surface. The separation gap associated with it will be called a step cavity or step orifice. Natural examples are shown by Hallet and Anderson [1980, Figure 3] and by Kamb and La Chapelle [1964, Figure 7]. The second type of bedrock feature is a wave, idealized as a sinusoid, with crest running transverse to ice flow. The separation gap that forms on the lee slope of such a wave form (Figure $4 b$ ) will be called a wave cavity or wave orifice. A natural example is given by Kamb and La Chapelle [1964, Figure 2]. The main distinctions between step cavities and wave cavities (or the corresponding orifices) are the following: (1) in a step cavity the ice separates from the bed at a sharp break in slope of the bedrock surface, whereas a wave cavity has no such predetermined point of ice separation, and (2) a wave cavity is inhibited from extending into the stoss slope downstream, whereas no such inhibition is presented to a step cavity. Because of the stress concentration at a sharp slope break, a step cavity will remain open at lower basal water pressure and lower sliding velocity than a wave cavity. Actual wavelike topographic features of glacier beds (roches moutonnées, more or less) often have a faceted lee slope that is a hybrid of the characteristics of a step and the rounded lee slope of a wave (Figure $4 c$ ); it will generate a leeside cavity that is rather like a step cavity at small amounts of separation and becomes like a wave cavity at large amounts of separation. Natural examples are shown by Carol [1947], Vivian and Bocquet [1973, Figures 2-4], and Vivian [1980, Figure 4]. One can also picture a rounded step (Figure 4d) that is a different type of hybrid; the cavity that forms behind such a rounded step will have the character of a wave cavity at small amounts of separation but will assume more and more the character of a step cavity as the amount of separation becomes large. For maximum clarity and distinctiveness, in the linked cavity model I will focus on the behavior of the pure or extreme types, the step cavity and the wave cavity. The transverse type of "N channel" considered qualitatively by Weertman [1972, Figure 10] and shown in Figure $4 e$ is in fact very similar to a wave cavity.

The idealized step of the model (Figure 5) is a rectangular step of height $h$, running transverse to the sliding direction. The resulting ice flow and separation problem is two-dimensional. A coordinate system $x, z$ is chosen with origin directly below the lip of the step, with $x$ running downstream parallel to the bed and $z$ perpendicular to the bed and upward (Figure $5 a$ ). The height of the separation gap is $g(x)$. The sliding-with-separation problem can be solved in closed form under the assumptions that the ice rheology is linear and that the slope of the sole, $g^{\prime}(x)$, is small so that the sliding ice constitutes a half-space, to an adequate approximation. (These are the same assumptions for which an exact solution of the basal sliding problem without separation is obtainable [Nye, 1969; Kamb, 1970, section 6].) Derivation of the solution is too lengthy to include here and will be given in a separate paper. The results needed for the linked cavity model are as follows. The gap height is

$$
g(x)=h\left(\frac{1}{2}-\frac{1}{\pi} \sin ^{-1} \frac{2 x-l}{l}-\frac{2(2 x-l) \sqrt{x(l-x)}}{\pi l^{2}}\right)
$$

where $0 \leq x \leq l$. The gap shape given by (4) is shown by the curve for $\Xi=0$ in Figure 8. The gap length (orifice breadth) is

$$
l=4 \sqrt{\frac{\eta v(h+m)}{\pi \sigma}}
$$


(a) WITHOUT ROOF MELTING

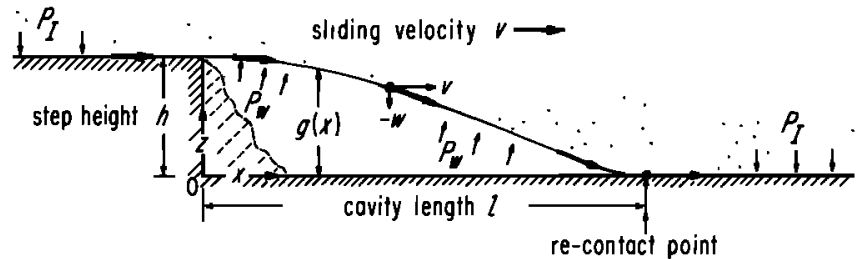

(b) WITH ROOF MELTING

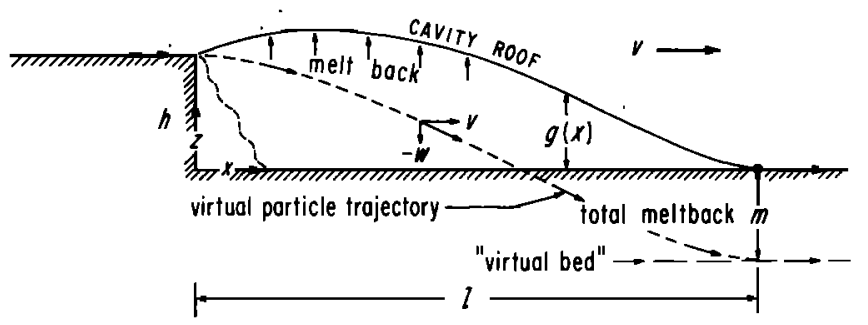

Fig. 5. Detailed geometry of ideal step cavity or step orifice (a) without and $(b)$ with ice roof melting by heating of basal water through viscous dissipation. The cavity is shown in vertical cross section parallel to the basal sliding direction. Ice is stippled, bedrock cross-hatched, cavity blank. The step is of height $h$. An $x, z$ coordinate system is taken with origin at the base of the step as shown. The gap height $g(x)$ is a function of longitudinal coordinate $x$. The cavity is filled with water at pressure $P_{\mathrm{W}}$, and the ice overburden pressure is $P_{\mathrm{l}}$. In sliding in the $x$ direction at velocity $v$, the ice separates from the bedrock at the lip of the step, $x=0$, and recontacts the bed at $x=l$. Here $w(x)$ is the $z$ component of ice motion at the cavity roof. In Figure $5 b$ the roof is melted back a total 2 distance $m$ as the ice traverses the length of the cavity. The path that an ice particle leaving the step lip would follow, moving with the ice mass, if it were not removed by melting is shown in Figure $5 b$ as a dashed line labeled "virtual particle trajectory"; it hits the "virtual bed," lowered by the distance $m$, at $x=l$

where $\eta$ is the ice viscosity, $v$ is the ice sliding velocity, $\sigma=P_{\mathrm{I}^{-}}$ $P_{\mathrm{W}}$ is the excess of ice overburden pressure $P_{\mathrm{I}}$ over basal water pressure $P_{\mathrm{W}}$ (i.e., the effective confining pressure), and $m=0$ if there is no melting of the gap roof. The rate at which ice flow tends to close the gap is given in terms of the $z$ component of flow velocity of the ice of the gap roof at each point and is

$$
w(x)=v g^{\prime}(x)=-\frac{\sigma}{2 \eta} \sqrt{x(l-x)}
$$

This closure rate is balanced by the effect of sliding in tending to open the gap, so that the gap is in a steady state.

According to (5), the gap length $l$ tends to 0 as $\sigma \rightarrow \infty$, but $l$ remains nonzero for any finite confining pressure $\sigma$, however large. Thus in the model, step orifices remain open to some extent even at very large $P_{\mathrm{I}}$ or very low $P_{\mathrm{W}}$. Under these extreme conditions, when $l \leq h$, the approximation $g^{\prime}(x) \ll 1$ breaks down, so that the relationships in (1)-(6) are not strictly valid, but it is assumed that they describe the model system to an adequate approximation up to the largest values of $\sigma$ that occur in practice.

Because actual ice rheology is nonlinear, (4)-(6) are only an approximation even when $g^{\prime}(x)$ is small. To deal with this, we use the simplest possible approach, which is to make $\eta$ shear-stressdependent in (5) and (6). Since all stresses would be hydrostatic if $\sigma=0$, it is logical to take $\sigma$ as the measure of shear stress level in the sliding-with-separation problem and hence in conformity with the standard nonlinear flow law to take

$$
\eta=\eta_{R}\left(\frac{\sigma_{R}}{\sigma}\right)^{n-1}
$$

where $\eta_{R}$ is the effective viscosity at a reference shear stress level
$\sigma_{R}$. These two quantities are in principle related by the ice flow law and the geometry of ice deformation in the sliding-withcavitation problem; $n=3$ will be assumed. The reasonableness of assumption (7) is shown by the fact that the formula giving the closure rate of a circular tunnel for linear rheology is converted by (7) to the exact result for nonlinear rheology [Nye, 1953] except for a numerical factor, which can be absorbed into $\eta_{R}$ or $\sigma_{R}$.

The shape of wave orifices will be considered in terms of separation in sliding over a "quasisinusoidal" bed with wave crests perpendicular to the sliding direction. It is based on a sine wave of wavelength $\lambda$ and half amplitude $a$. The separation geometry is shown in Figure 6a. The length of the separation gap is $l$, and the gap height is $g(x)$, the origin for $x$ being at the head of the gap (point of ice-bedrock separation), the position of which is not prescribed in advance. The two-dimensional sliding-withseparation problem for linear rheology can be solved in closed form if $a \ll \lambda$, as is appropriate for wave orifices which are narrow separation gaps. The normal stress across the ice-bedrock contact before onset of separation is assumed to vary as $\left(x-x_{0}\right)^{2}$, the amplitude of the variation being matched to that for the sinusoidal bedrock wave form in the vicinity of its inflection point, located at $x_{0}$. The actual bedrock wave form that gives this parabolic normal stress variation in the $x$ interval over which separation later occurs is here called a "quasisinusoidal" waveform. For a strictly sinusoidal waveform a restriction $l$ \& $\lambda$ on the validity of the sliding-with-cavitation solution would apply, but for a quasisinusoidal waveform as just defined, it does not. The method of solution is fundamentally the same as for step cavities but is more involved because of the complication caused by the initially unknown location of the separation point. The lengthy derivation will be given in a separate paper, where its relation to

\section{(a) WITHOUT ROOF MELTING}

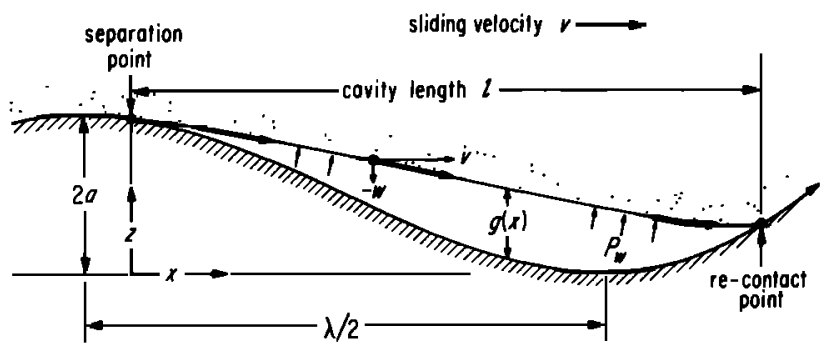

(b) WITH ROOF MELTING

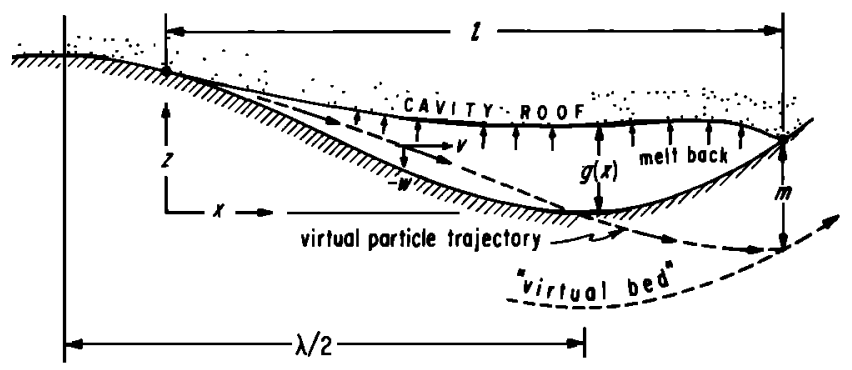

Fig. 6. Detailed geometry of ideal wave cavity or wave orifice (a) without and $(b)$ with ice roof melting. Conventions and symbols are as in Figure 5. The bedrock wave has wavelength $\lambda$ and half amplitude $a$. The origin of the $x, z$ coordinate system is taken directly below the ice-bedrock separation point; the $x$ axis parallels the mean bed slope of the wave. Positions of the separation point and recontact point are determined in the sliding-with-separation solution. Both points move to different positions when there is roof melting (Figure $6 b$ ). 
the different solutions by Lliboutry [1968] and Fowler [1986] for the same problem will be pointed out. The results needed here are as follows. The gap length is

$$
l=\frac{4 \lambda}{\pi}\left(\frac{2}{5}\right)^{\frac{1}{2}}\left(1-\frac{\sigma}{\Sigma}\right)^{\frac{1}{2}}, \sigma \leq \Sigma
$$

where, as before, $\sigma=P_{1}-P_{\mathrm{W}}$ and where

$$
\Sigma=8 \pi^{2} \eta a v / \lambda^{2}
$$

$\Sigma$ is called the wave cavitation parameter. It is the limiting effective confining pressure for cavitation: for $\sigma \geq \Sigma$ there is no separation. Thus, in contradistinction to step cavities, wave cavities form only for a sufficiently high water pressure $P_{\mathrm{w}}$, such that $P_{\mathrm{W}}>P_{\mathrm{I}}-\Sigma$. The gap height is given by

$$
g(x)=\frac{2 \pi^{4}}{3} \frac{a}{\lambda^{4}} x^{5 / 2}(l-x)^{3 / 2}
$$

for $0 \leq x \leq l$. The separation point lies a distance (3/8) $l$ upstream from the inflection point on the lee slope of the sine wave. The shape of the separation gap is shown by the curve for $\Xi^{\prime}=0$ in Figure 10.

Nonlinear rheology can be introduced in the way done by $K a m b[1970$, p. 693]. The strain rate dependence of $\eta$ is written

$$
\eta=N \dot{\varepsilon}^{-1+\frac{1}{n}}
$$

where $N$ and $n$ are constants and where $\dot{\varepsilon}$ is the second strain rate invariant. We will take $n=3$ and $N=0.94$ bar $\mathrm{yr}^{1 / 3}$. For sliding over a single sine wave of wavelength $\lambda$, the value of $\dot{\varepsilon}$ at a height $\lambda 2 \pi$ above the sliding interface, which is assumed to govern the effective viscosity as discussed by Kamb [1970, p. 694], is

$$
\dot{\varepsilon}=4 \pi^{2} e^{-1} a \nu \lambda^{-2}
$$

as can be obtained from equations (47) and (49) of Kamb [1970] with the assumption $\lambda$ \& $\lambda_{\mathrm{o}}$ (the transition wavelength) so that regelation is negligible. Hence from (11), with $n=3$,

$$
\eta=N\left(\frac{e \lambda^{2}}{4 \pi^{2} a v}\right)^{\frac{2}{3}}
$$

Introducing this into (9), we obtain

$$
\Sigma=2 N\left(\frac{4 \pi^{2} e^{2} a v}{\lambda^{2}}\right)^{\frac{1}{3}}
$$

showing that the wave cavitation parameter increases with the sliding velocity as $v^{1 / 3}$. The fact that the quantity $\sigma$ does not enter into what determines $\eta$ in (12) indicates that the treatment is an approximation in which the effect of the additional stresses associated with the cavitation is neglected. This can be done as a reasonable approximation here because $\sigma<\Sigma$, whereas for the step cavity, in (7), $\eta$ must depend on $\sigma$, as in tunnel closure, there being no ambient stress level analogous to $\Sigma$.

\section{EFFECT OF HEAT DISSIPATION ON CAVITATION AND WATER FLUX}

When there is meltback of the cavity roof, amounting to a total meltback distance $m$ as the ice of the roof traverses the cavity from point of separation to point of recontact, it can be taken into account in the solution of the sliding-with-separation problem discussed in section 6 by the simple artifice of requiring the ice to recontact the bed at a point lower by a distance $m$ than if there were no meltback. This is indicated in Figures $5 b$ and $6 b$. where the cavity roof with meltback is drawn with a solid line, while the dashed line shows the imaginary ("virtual") trajectory that an ice particle leaving the separation point would follow, moving with the ice mass, if the sole were not melted back and if the bed downstream from the separation point were lowered a moving with the ice mass, if the sole were not melted back and if the bed downstream from the separation point were lowered a distance $m$. For the step cavity, the height of the "virtual" step in this case is $h+m$, which explains the appearance of this quantity in (5), giving the gap length $l$ for meltback $m$.

To obtain the steady state gap profile $g(x)$ for a step cavity with meltback, the meltback rate (3) is put in opposition to the gap closure rate (6) to give the progressive widening or narrowing of the gap as seen moving with the ice:

$$
v g^{\prime}(x)=\dot{m}(x)+w(x)
$$

When (3) and (6) are introduced into (14), after first eliminating $\sigma$ from (6) with the use of (5), and when the variables are nondimensionalized by defining

$$
\gamma=g / h \quad \xi=x / l \quad \mu=m / h
$$

one obtains the dimensionless differential equation

$$
\frac{d \gamma}{d \xi}=2 \Xi \sqrt{(1+\mu)} \gamma^{5 / 3}-\frac{8}{\pi}(1+\mu) \sqrt{\xi(1-\xi)}
$$

where

$$
\Xi=\frac{2^{1 / 3}}{\pi^{1 / 2}} \frac{(\alpha \Lambda \omega)^{3 / 2}}{D M}\left(\frac{\eta}{\nu \sigma}\right)^{\frac{1}{2}} h^{\frac{7}{6}}
$$

The dimensionless quantity $\Xi$ will be called the orifice meltingstability parameter, for reasons that appear later (section 8). It provides a measure of the importance of roof melting by viscous dissipation in the linked cavity system.

The gap profile $\gamma(\xi)$ is obtained by integrating (16), starting at $\chi(0)=+1$. For a given value of $\Xi$, there will be a value of $\mu$ such that when (16) is integrated from $\xi=0$ to 1 , one obtains $\gamma(1)=0$. This is the condition for the ice to recontact the bed at $\xi=1$, as required; hence it gives the nondimensionalized meltback $\mu$ that corresponds to the given value of $\Xi$. The steady state gap profile $\gamma(\xi)$ corresponding to $\Xi$ is given by the integral of (16) for the value of $\mu$ so determined. The results of numerical integration of (16) for a succession of values of $\Xi$ from 0 to 1.1 are shown in Figures 7 and 8 . Figure 7 gives the meltback quantity $\mu$ as a function of $\Xi$, and Figure 8 gives the nondimensionalized gap profiles $\chi(\xi)$ for several values of $\Xi$. The amount of meltback $m$ is directly seen from the curve of $\mu=m / h$ in Figure 7 . For $\Xi \sim 1$, $m$ becomes rather large, considerably larger than the step height $h$. 


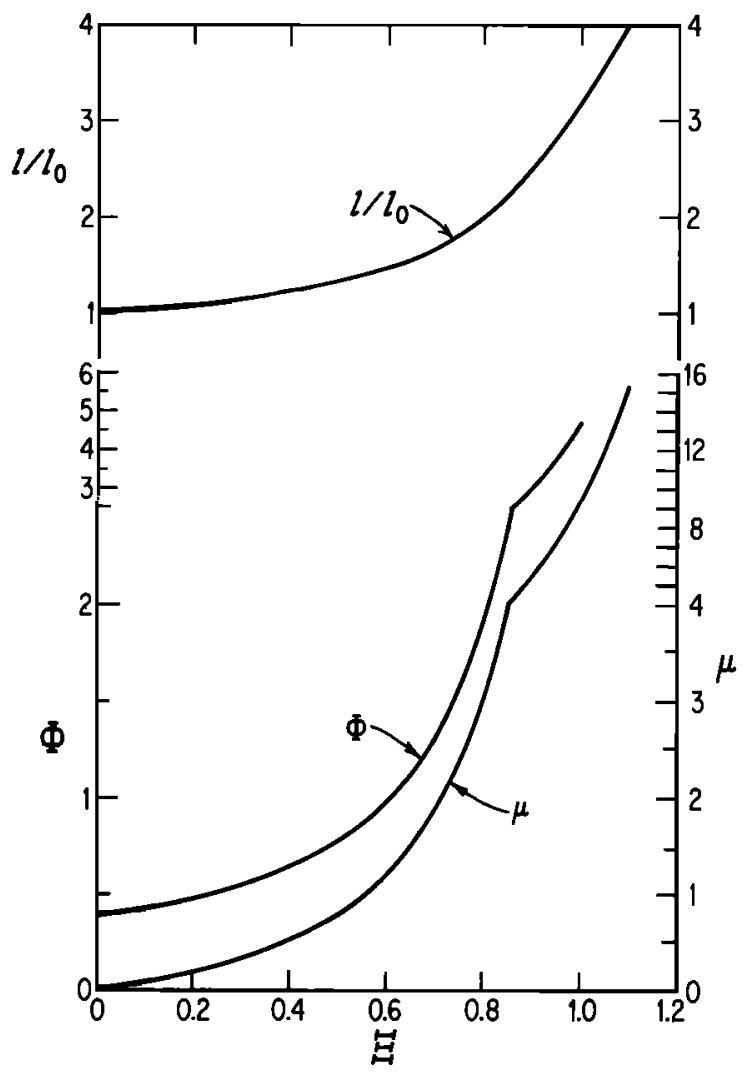

Fig. 7. Step orifice model: dependence of dimensionless parameters on the melting-stability parameter $\Xi$, whose definition is given in (17). Here $\mu$ is the meltback parameter $m / h$, determined from (16) as described in the text, $l / l_{0}$ is the orifice gap elongation relative to the gap length $l_{0}$ for no roof melting ( $\Xi=0$ ), and $\Phi$ is the flux factor defined in (19) and calculated from the results of integrating (16), given in Figure 8. Note that there is a scale change for the $\mu(\Xi)$ curve at $\mu=4$ and for the $\Phi(\Xi)$ curve at $\Phi=2.5$.

Figure 8 shows how the shape of the gap profile responds to the meltback. The roof is raised, and the peak of the roof is shifted downstream as melting increases. The biggest effect, however, is the lengthening of the gap, which is not shown in Figure 8 because of the scaling of $\xi$. It can be seen in terms of the ratio $l / l_{0}$, given in Figure $7 ; l_{0}$ is the gap length for no meltback $(\Xi=0, \mu=0)$, given by (5) for $m=0$; thus $l / l_{0}=\sqrt{1+\mu}$. The lengthening of the gap when $\Xi \approx 1$ is by a factor of about 3 .

Once the gap profile is determined, the water flux carried by the system can be found from (3). The result can be expressed as follows:

$$
Q_{\mathrm{w}}=\frac{2^{4 / 3}}{\pi^{1 / 2}} \frac{N_{\mathrm{o}}}{M}\left(\frac{\alpha \Lambda}{\omega}\right)^{\frac{1}{2}}\left(\frac{\eta v}{\sigma}\right)^{\frac{1}{2}} h^{\frac{13}{6}} \Phi
$$

where

$$
\Phi=\sqrt{1+\mu} \int_{0}^{1} \gamma^{5 / 3} d \xi
$$

The "flux factor" $\Phi$, obtained by numerical integration of the results in Figure 8, is given as a function of $\Xi$ in Figure 7.

The steady state gap profile for a wave orifice with meltback is obtained from (13) in the same basic way, as suggested by Figure $6 b$, with the appropriate $w(x)$, related to (10). The form of $w(x)$ is complicated by the fact that the positions of both the separation point and the recontact point are affected by the meltback. I will pass over the somewhat involved details and simply give the results. If the nondimensionalization is done by $\xi=x / l$ and $\gamma=g / g_{0}$, where

$$
\frac{g_{0}}{a}=\left(\frac{128}{75}\right)\left(1-\frac{\sigma}{\Sigma}\right)^{2}
$$

and if we define a "meltback parameter"

$$
v=\frac{2^{4}}{\pi^{5}}\left(\frac{\lambda}{T}\right)^{4} \frac{m}{a}
$$

and the dimensionless quantity

$$
B=\frac{5}{\left(5-2 v+v^{2}\right)}
$$

then the differential equation for the gap profile can be written

$$
\frac{d \gamma}{d \xi}=\Xi^{\prime}(5 B)^{1 / 2} \gamma^{5 / 3}-B^{2} \xi^{3 / 2}(1-\xi)(8 \xi+3 v-5)
$$

The melting-stability parameter $\Xi^{\prime}$ that arises in (23) is

$$
\Xi^{\prime}=\frac{2^{7}}{3^{2 / 3} 5^{7 / 3}} \frac{(\alpha \Lambda / \omega)^{3 / 2}}{D M}\left(\frac{\eta}{v \Sigma}\right)^{\frac{1}{2}} a^{\frac{7}{6}}\left(1-\frac{\sigma}{\Sigma}\right)^{\frac{11}{6}}
$$

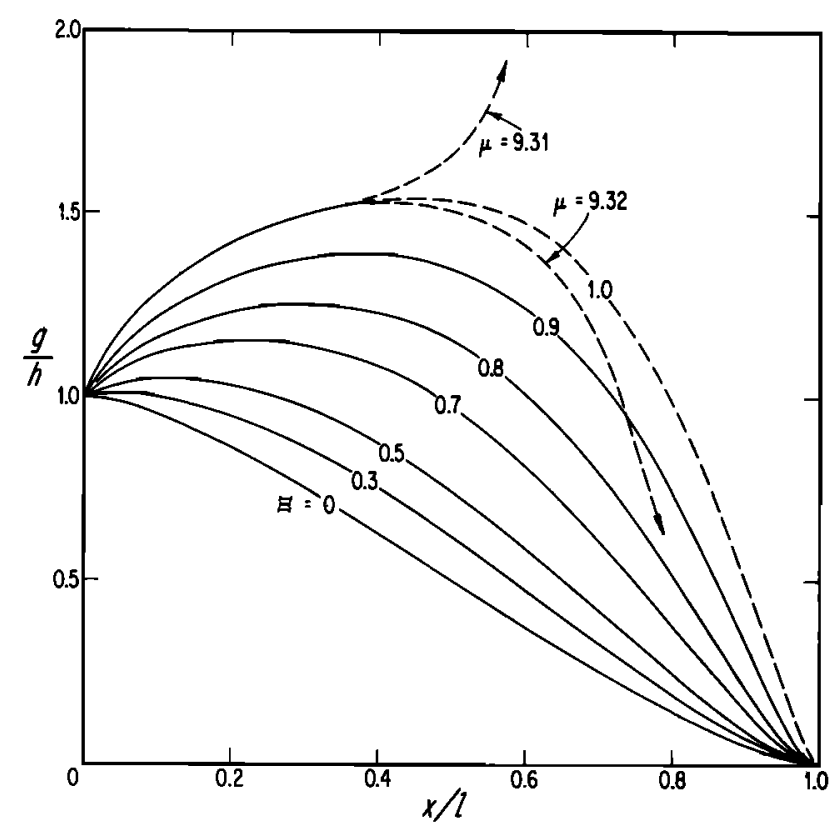

Fig. 8. Steady state configuration of the step orifice roof for values of the melting-stability parameter $\Xi$ from 0 to 1.0 . For each value of $\Xi$, the gap height $g(x)$ is shown in terms of $g / h$ as a function of dimensionless longitudinal coordinate $x / l$, where $l$ is the gap length. (The $l$ varies with $\Xi$ as indicated by the $l / l_{0}$ curve in Figure 7 .) The curves are obtained by integration of (16) with the appropriate $\mu$ values as explained in the text. At $\Xi=1$, integration of (16) for the two indicated values of $\mu$ gives two widely diverging curves $g(x)$ as shown by the dashed curves with arrows. 


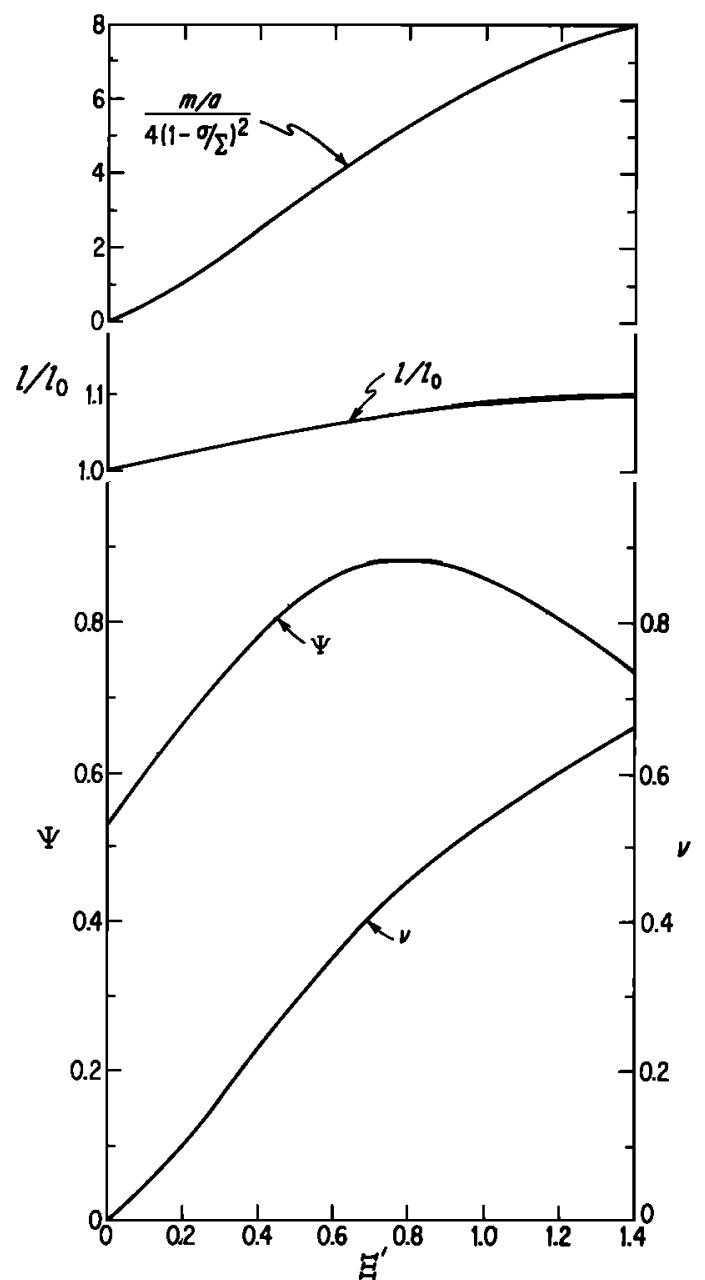

Fig. 9. Wave orifice model: dependence of dimensionless parameters on the melting-stability parameter $\Xi^{\prime}$, defined in (24) or (25). Here $v$ is the meltback parameter defined in terms of $\mathrm{m} / \mathrm{a}$ in $(21)$ and calculated from (23) as explained in the text, and $l / l_{0}$ is the relative orifice gap elongation, from $l / l_{0}=B^{1 / 2}$, where $B$ is defined in (22). The quantity $(m / a) / 4(1-\sigma / \Sigma)^{2}$ gives a measure of the roof meltback from (27). $\Psi$ is the flux factor defined in (30) and calculated from the results of integrating (23), given in Figure 10.

As given in (24), it has a form similar to $\Xi$ in (17), differing mainly in the way $\sigma$ and $\Sigma$ enter. However, $\Sigma / \eta$ in (24) can be eliminated by substitution from (9), and the parameter $\Xi^{\prime}$ then assumes a less similar form:

$$
\Xi^{\prime}=\frac{2^{11 / 2}}{3^{2 / 3} 5^{7 / 3} \pi} \frac{(\alpha \Lambda \omega)^{3 / 2}}{D M} \frac{\lambda a^{2 / 3}}{v}\left(1-\frac{\sigma}{\Sigma}\right)^{\frac{11}{6}}
$$

in which, notably, the effective viscosity $\eta$ does not appear.

The integration of (23) is done in the same basic way as (16): for a given $\Xi^{\prime}$, a value of the parameter $v$ is sought such that when (23) is integrated from $\xi=0$ to 1 , starting at $\chi(0)=0$, one arrives at $\gamma(1)=0$. Results of this procedure are given in Figures 9 and 10.

Figure 9 shows the meltback parameter $v$ as a function of $\Xi^{\prime}$. The actual meltback amount $m$ is obtained by solving (21) for $m$ and introducing $v$ from Figure 9 and the cavity length $l$ from

$$
\frac{l}{\lambda}=\frac{4}{\pi}\left(\frac{2 B}{5}\right)^{\frac{1}{2}}\left(1-\frac{\sigma}{\Sigma}\right)^{\frac{1}{2}}
$$

of which (8) is the special case for $v=0$ (no meltback). Thus

$$
\frac{m}{a}=\frac{64 \pi v}{\left(5-2 v+v^{2}\right)^{2}}\left(1-\frac{\sigma}{\Sigma}\right)^{2}
$$

The meltback ratio $m / a$ depends on $\Xi^{\prime}$ via $v$ and in addition on $\sigma / \Sigma$ via the last factor in (27). Figure 9 gives a curve of the quantity $(m / a)(1-\sigma / \Sigma)^{-2} 2^{-2}$, which depends on $\sigma$ only via the dependence of $\Xi^{\prime}$ on $\sigma$ and is equal to $m / a$ for $\sigma / \Sigma=1 / 2$. The large indicated amount of meltback for $\Xi^{\prime} \sim 1$ is striking, as it is also for step orifices.

Figure 10 shows wave-orifice gap profiles $\chi(\xi)$ for a succession of $\Xi^{\prime}$ values. The gap height, plotted as the dimensionless ratio $g / g_{0}$ in Figure 10 , is scaled by the dimension $g_{0}$ given in (20), which is the midpoint height that the gap would have if there were no melting (value of $g$ in (10) at $x=l / 2$ ). The gap height in Figure 10 is of course greatly exaggerated in relation to the gap length because of the way the plot is made. The gap length is obtained from $l / l_{0}=\sqrt{B}$, based on (26). As the curve of $l / l_{0}$ versus $\Xi^{\prime}$ in Figure 9 indicates, the gap length changes little with increasing meltback, contrary to the large effect for step orifices shown by the $l / l_{0}$ curve in Figure 7 .

A surprising feature of the gap profiles is the effect of meltback in causing a sagging of the roof in the upstream part of the slope cavity, seen by comparing the $\Xi^{\prime}=0$ and $\Xi^{\prime}=1$ curves in Figure 10 , for $x / l<0.5$. The sagging appears to be linked to the fact that the point of separation shifts downstream as meltback increases. The shift, which is not depicted in Figure 10 because the origin is at the separation point, is indicated by the following relation between meltback parameter $v$ and the distance $x_{0}$ from the separation point to the inflection point:

$$
x_{0}=\frac{3-v}{8} l
$$

The water discharge $Q_{w}$ carried by the linked cavity model

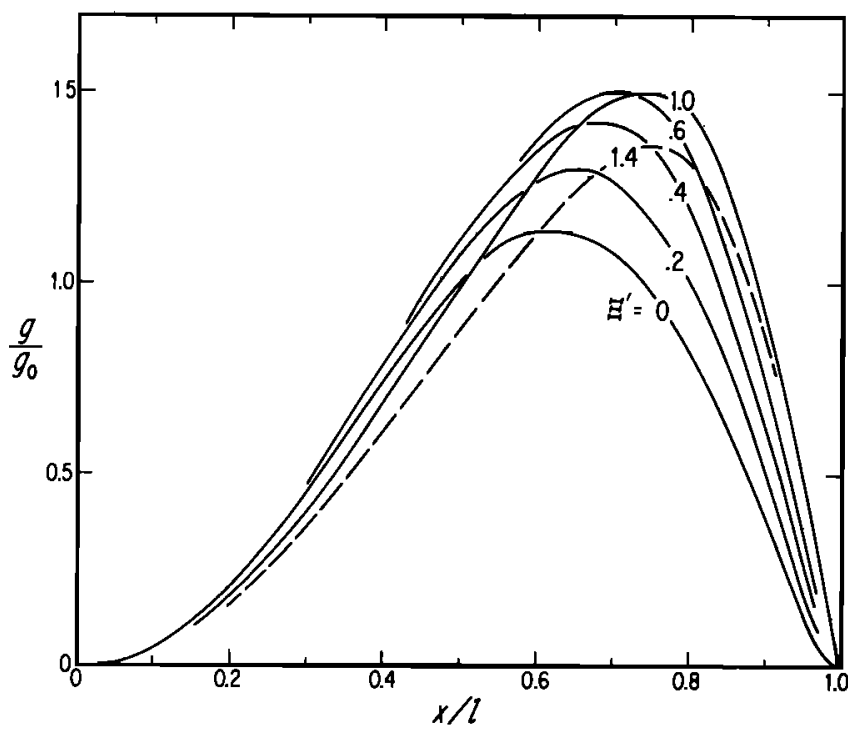

Fig. 10. Steady state configuration of the wave orifice gap for values of the melting-stability parameter $\Xi^{\prime}$ from 0 to 1.4 . For each value of $\Xi^{\prime}$, the gap height $g(x)$ is shown in terms of the ratio $g / g_{0}$, where $g_{0}$ is the height midway along the length of the gap $(x=l / 2)$ in the absence of roof melting $\left(\Xi^{\prime}=0\right)$, given by $(20)$. The longitudinal coordinate $x$ is scaled by the gap length $l$, given by (26). The curves are obtained by integration of (23) with the appropriate values of $v$ as explained in the text. 
with wave cavity type orifices, from (2), (20), and (26), can be written

$$
Q_{\mathrm{W}}=\frac{2^{25 / 2}}{75^{5 / 3} \pi} \frac{N_{\mathrm{o}}}{M}\left(\frac{\alpha \Lambda}{\omega}\right)^{\frac{1}{2}} \lambda a^{\frac{5}{3}}\left(1-\frac{\sigma}{\Sigma}\right)^{\frac{23}{6}} \Psi
$$

where

$$
\Psi=B^{1 / 2} \int_{0}^{1} \gamma^{5 / 3} d \xi
$$

is a "flux factor" analogous to $\Phi$ in (19). A curve of $\Psi$ versus $\Xi$ ', obtained from the results in Figure 10, is given in Figure 9.

\section{ORIFICE STABILITY}

In the integrations leading to the curves in Figure 8, it is found that as $\Xi$ increases to near 1 , the curves become very sensitive to the value of $\mu$. This is illustrated by the two dashed curves (with arrows) for $\Xi=1$ in Figure 8, obtained by integrating with $\mu=9.31$ and $\mu=9.32$, respectively. Under these circumstances it becomes difficult to find the value $\mu$ that permits the condition $\gamma(1)=0$ to be satisfied, and for this reason the $\Xi=1$ curve in Figure 8 is shown dashed beyond where the calculated curves diverge. A similar thing happens in the integrations leading to the curves in Figure 10 when $\Xi^{\prime}$ increases to about 1.5. Thus, for $\Xi^{\prime}=1.5$ the curve $\gamma(\xi)$ calculated by integrating with $v=0.679$ diverges to $\gamma>5.4$ for $\xi>0.8$, while the curve with $v=0.680$ drops to $\gamma=-0.25$ at $\xi=1.0$. This behavior appears to be the manifestation of an orifice instability that commences at critical parameter values $\Xi \sim 1$ or $\Xi^{\prime} \sim 1.5$.

To shed light on this instability, it is helpful to analyze the transient behavior of a step orifice subjected to a perturbation in water pressure. We start with a step orifice in steady state, under effective confining pressure $\sigma_{0}$, with meltback parameters $\Xi_{0}$ and $\mu_{0}$ and initial gap length $l_{0}$. The effective confining pressure is then abruptly decreased to $\sigma<\sigma_{0}$, so that the orifice begins to enlarge. We follow the transient response in terms of what happens to a reference point that starts at the lip of the step at the moment the perturbation is applied. The gap length $l$ will now be a function of time or, equivalently, a function of the position $\xi=x / l_{0}$ of the reference point as it moves forward with the ice. From the way equation (16) was constructed, one can see that with two modifications it can be used to determine the gap height $\gamma(\xi)=g(\xi) / h$ that develops at the position $\xi$ of the reference point as it moves along:

$$
\frac{d \gamma}{d \xi}=2 \Xi_{0} \sqrt{1+\mu_{0}} \gamma^{5 / 3}-\frac{8}{\pi}\left(1+\mu_{0}\right) \frac{\sigma}{\sigma_{0}} \sqrt{\xi(\gamma(\xi)-\xi)}
$$

The ratio $\sigma / \sigma_{0}$ appears in the right-hand term of (31) because the gap closure rates are proportional to $\sigma$, as (6) indicates. The appearance of $\Upsilon(\xi)=l / l_{0}$ in the same term derives from the $l$ in (6), which now varies with $\xi$ as just explained. To find out how $\gamma$ at the reference point varies with position $\xi$ as it moves along, we want to integrate (31), starting at $\gamma(0)=1, \Upsilon(0)=1$. To do this, we need to know the function $\mathrm{r}(\xi)$, describing the motion of the recontact point with time $t$ (or equivalent $\xi=v t / l_{\mathrm{o}}$ ).

The rigorous determination of $\mathrm{r}(\xi)$ is a complicated problem, beyond the scope of this paper. A detailed treatment, to be given elsewhere, yields two conclusions of importance here: (1) at the instant of the pressure perturbation, the orifice gap immediately begins to lengthen at a rate $\dot{l}(0)=v\left(1-\sigma / \sigma_{0}\right)$, and (2) the maximum rate of gap lengthening at any time is $v$, that is, $i \leq v$. Because we are concerned with the possibility of an unstable orifice enlargement consequent upon increase in water pressure and because maximum stabilization of the orifice against enlargement is provided by maximum closure rate and therefore maximum length $l$, as indicated by (6), conclusion 2 makes the following simple procedure valid as a test for the existence of an instability. We make the approximation that $i$ is constant, $i=f v$, so that

$$
\Upsilon(\xi)=1+f \xi
$$

For any particular value of $\Xi_{0}$, we try to choose the proportionality constant $f$ such that when (31) is integrated forward from $\xi=0, \gamma(0)=1$, the reference point hits the bed (that is, $\gamma(\xi)$ goes to zero) at a "touchdown" point $\xi_{\mathrm{T}}$ compatible with the $i$ value chosen, which requires $\Upsilon\left(\xi_{\mathrm{T}}\right)=\xi_{\mathrm{T}}$, or, from (32),

$$
f=1-\xi_{\mathbf{T}}^{-1}
$$

If a value $f<1$ can be found such that these conditions are met, then a stable transient response of the orifice is possible. If, on the other hand, with the choice $f=1$ in (32) the integration of (31) leads to $\gamma(\xi)$ increasing without bound, then it is impossible for the orifice to have a stable transient response because $f=1$ gives the maximum possible closure rate for the orifice at all stages (conclusion 2 above).

Results of the above procedure are shown in Figure 11 in terms of trajectories $\gamma(\xi)$ of reference points for an assortment of perturbations at several values of $\Xi_{0}$. The magnitude of the perturbation for each curve is measured by the value of $1-\sigma / \sigma_{0}$ and is indicated by the $\sigma / \sigma_{0}$ values in Figure 11; the $f$ value used in calculating each curve is also given. Figure 11 shows that for perturbations as large as $1-\sigma / \sigma_{0}=0.2$, the transient response of the orifice can be stable for $\Xi_{0}$ as large as 0.7 , but for $\Xi_{0}=0.8$, the perturbation $1-\sigma / \sigma_{0}=0.2$ leads to an unstable response, the orifice enlarging without bound. For $\Xi_{0}=0.9$, a perturbation of only $10 \%\left(\sigma / \sigma_{0}=0.9\right)$ is sufficient to cause an unstable response. These results indicate that step orifices have a runaway instability for $\Xi_{0} \geq 1$. This stability limit is probably somewhat overestimated because near the limit, as $f \rightarrow 1$, (32) overestimates $r$, at least near $\xi=0$, where the assumed motion $i \rightarrow$ is larger than allowed by conclusion 1 stated in the previous paragraph.

It seems very likely that the same type of instability arises for wave orifices for $\Xi^{\prime} \geq 1.5$, as suggested by the parallelism in the behavior of the integration for determining the steady state profiles of wave orifices and step orifices, discussed earlier.

The cause of the instability can be linked to the increase in melting rate that occurs when the gap height increases. The instability is demonstrated in Figure 11 under perturbations of $\sigma$, but there is little doubt that it would appear equally under perturbations in $\gamma(\xi)$ itself. A closely related type of instability is a feature of ice tunnels, as discussed in section 11.

The foregoing calculation, in combination with conclusion 1 stated above, indicates that the response of the step orifice system to pressure perturbations is definitely stable if $\Xi$ and the pressure perturbation are small enough. If the gap lengthening rate $i=f v$ used in the calculation of a given transient response curve in Figure 11 (as formulated in (32)) is less than the initial lengthen- 


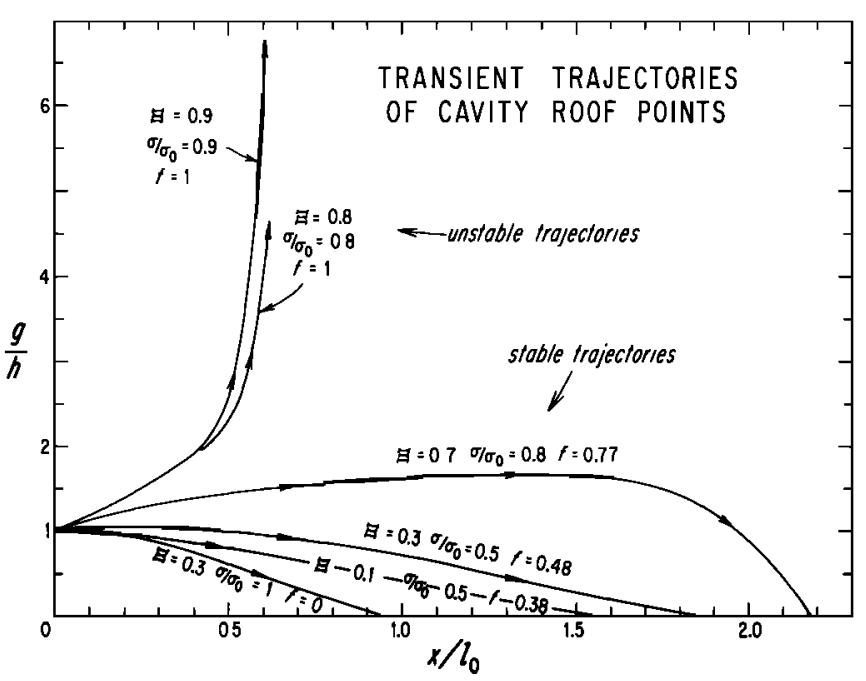

Fig. 11. Transient trajectories of a "reference point" that follows the step orifice roof upon perturbation of the system from the steady state by a sudden increase in basal water pressure for various initial and perturbed conditions. The curves are calculated from (31)-(33) as explained in the text; the parameters used in calculating each curve are given alongside. Here $\sigma_{\mathrm{o}}$ is the initial effective confining pressure $P_{\mathbf{I}}-\boldsymbol{P}_{\mathrm{W}}$ prior to the perturbation, $\sigma$ the perturbed value $\left(\sigma<\sigma_{0}\right) ; \Xi_{0}$ is the initial value, prior to perturbation, of the melting-stability parameter $\Xi$ in (17); and $f$ is the constant in (32) and (33), which is required to be $\leq 1$ (see text). The coordinate $x$ is the longitudinal position of the reference point, which starts at the step lip $(x=0$, where $g=h)$ at the moment of perturbation and increases with time at the sliding speed $v_{;}$it is scaled by $l_{0}$, the initial steady state cavity length. For stable trajectories, in which the reference point ultimately makes contact with the bed $(g=0)$, the dimensionless coordinate $x / l_{0}$ of the recontact point is $\xi_{\mathrm{T}}$ in (33). Trajectories that diverge upward with $f=1$ represent unstable responses to the imposed pressure perturbation.

ing rate $l(0)=v\left(1-\sigma / \sigma_{0}\right)$ required by conclusion 1 , then it is quite likely that in the response of the actual physical system $\Upsilon(\xi)$ will always be larger than the $\Upsilon(\xi)$ used in the calculation, which is based on the assumption of constant $i$. The larger $\Upsilon$ corresponds to a faster roof collapse rate (from (6)) and therefore a stronger assurance that the gap does not go into runaway enlargement. Thus a stable response is definitely indicated if $f<1-\sigma / \sigma_{0}$. This condition is satisfied by the curves for $\Xi_{0}=0.1$ and 0.3 in Figure 11. Clearly a stable response will also be obtained for any perturbation smaller than the one $\left(1-\sigma / \sigma_{0}=0.5\right)$ used in calculating these curves. The curve for $\Xi_{0}=0.7$ appears also to indicate a stable response, but this is not firmly established because the above condition is not satisfied in this case. The behavior of the steady-state-profile integrations gives the impression that both the step-orifice and wave-orifice systems have an appreciable degree of stability for $\Xi$ or $\Xi^{\prime}$ up to about 0.8 . Stability will be increased in an actual linked cavity system by transfer of some of the heat of viscous dissipation to the roofs of the large cavities, with release of the assumption of local heat transfer (section 5).

\section{QUANTITATIVE EVALUATION OF MODEL}

The hydraulic performance of the linked cavity model is shown in Figure 12 in terms of curves of water discharge $Q_{w}$ versus basal water pressure $P_{\mathrm{W}}$ or effective confining pressure $\sigma=P_{\mathrm{I}}-P_{\mathrm{W}}$. For ready comparison with the borehole water level observations from Variegated Glacier, $P_{W}$ is represented in
Figure 12 in terms of water level depth in an ice mass $400 \mathrm{~m}$ thick. Separate curves are given for a linked cavity system with step orifices and for one with wave orifices.

The $Q_{\mathrm{W}}$ versus $\sigma$ curve for the step orifice linked cavity model in Figure 12 is calculated from (18) with the following parameter values and with nonlinear ice rheology taken into account by (7):

$\begin{array}{ll}D=31 \mathrm{~km} & \rho_{\mathrm{I}} H / \rho_{\mathrm{w}} g_{\mathrm{r}} \\ \alpha=0.1 & \text { longitudinal hydraulic gradient } \\ \Lambda=10 & \text { head gradient concentration factor }\left(L_{\mathrm{c}} / L_{\mathrm{o}}\right) \\ \omega=4 & \text { conduit system tortuosity } \\ N_{\mathrm{o}}=50 & \text { orifice number } \\ M=0.1 \mathrm{~m}^{-1 / 3} \mathrm{~S} & \text { Manning roughness } \\ \eta_{\mathrm{R}}=0.1 \mathrm{bar} \text { yr } & \text { reference ice viscosity } \\ \sigma_{\mathrm{R}}=6 \mathrm{bars} & \text { reference effective pressure } \\ h=5 \mathrm{~cm} & \text { step height }\end{array}$

The system parameters $\Lambda, \omega, N_{0}$, and $h$ are chosen arbitrarily but with the intent that they be representative of what one might expect in a linked cavity system at the bed of Variegated Glacier in surge, with surface slope $\alpha \cong 0.1 . N_{0}=50$ means one orifice every $20 \mathrm{~m}$, on average, across the $1-\mathrm{km}$ width of the glacier. $\Lambda=10$ means that most of the length of the water flow paths is in

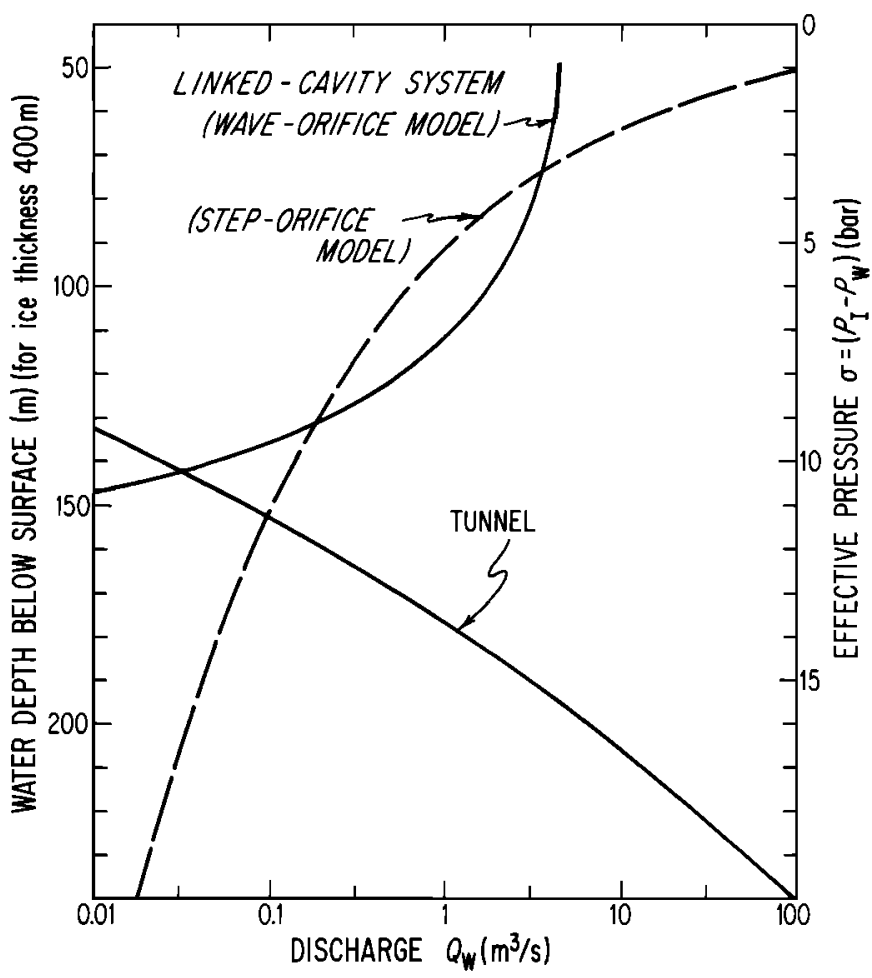

Fig. 12. Results of the linked cavity model and comparison with the tunnel model for parameter values given in section 9 . The effective confining pressure $\sigma=P_{\mathrm{I}}-P_{\mathrm{W}}$ (ice overburden pressure minus basal water pressure) is plotted against discharge $Q_{\mathrm{W}}$ carried by the basal water conduit system for two types of linked cavity model (with step orifices and wave orifices) and for the tunnel model. On the left, $P_{w}$ is represented in terms of borehole water level in ice $400 \mathrm{~m}$ thick, for ready comparison with data from Variegated Glacier in and out of surge. The step orifice curve is from (38b), the wave orifice curve from (42b), and the tunnel curve from (43). The melting-stability parameter for the step orifice curve is $\Xi=0.18$, from (37), and for the wave orifice curve, $\Xi^{\prime} \leq 0.20$, from $(41 b)$. Dimensional features associated with these curves are discussed in section 9 . 
cavities, only a tenth of the length being through orifices. The ratio 10 is thought reasonable, perhaps minimal, for a linked cavity system when there is very extensive cavitation over the bed, with small orifices throttling the flow, as visualized in Figure 1. The Manning roughness value is one that has been thought appropriate in application to glacier tunnels [Röthlisberger, 1972, p. 181; Clarke et al., 1984, pp. 292 and 295]. The tortuosity is chosen somewhat larger than the value $\omega=3$ thought appropriate for glacier tunnels by Brugman [1986, p. 219]. The $\eta_{R}$ value is what is calculated from the flow law (11) if we assume that the second stress invariant is equal to $\sigma_{R}=6$ bars.

In order to calculate the $Q_{\mathrm{W}}$-versus- $\sigma$ curve it is necessary to specify the sliding velocity $\nu$ as a function of $\sigma$, since it enters (18) both directly and through the flux factor $\Phi$ via its dependence on $\Xi$, which varies with $v$ as shown in (17). Although the relation between $v$ and $\sigma$ (dependence of sliding velocity on basal water pressure) can in principle be obtained from the mechanical theory of sliding with cavitation on which the foregoing treatment is based (or by the method of Fowler [1986]), for the evaluation here of the linked cavity model I use a simple empirical relation of the type

$$
\nu=v_{\mathrm{R}}\left(\frac{\tau}{\tau_{\mathrm{R}}}\right)^{p}\left(\frac{\sigma_{\mathrm{R}}}{\sigma}\right)^{q}={ }_{p} k_{q} \tau^{p} \sigma^{-q}
$$

where $\tau$ is the basal shear stress and $\nu_{R}$ is the sliding velocity under reference conditions of shear stress $\tau_{R}$ and effective pressure $\sigma_{\mathrm{R}}$; the independent constants in (34) are $p, q$, and the product ${ }_{p} k_{q}=v_{\mathrm{R}} \tau_{\mathrm{R}}{ }^{-p} \sigma_{\mathrm{R}}{ }^{q}$. The inclusion of a basal shear stress dependence in (34) is not needed for the immediate purpose at hand, for which we will take $\tau=\tau_{R}$, but is needed later (section 12). On the basis of laboratory experiments on the sliding of ice over rock surfaces, Budd et al. [1979, p. 164] proposed (34) with $p=3$ and $q=1$ :

$$
v=v_{\mathrm{R}}\left(\frac{\tau}{\tau_{\mathrm{R}}}\right)^{3} \frac{\sigma_{\mathrm{R}}}{\sigma}={ }_{3} k_{1} \frac{\tau^{3}}{\sigma}
$$

Bindschadler [1983] got an approximate fit to flow data for Variegated Glacier in the nonsurging state by taking (35) with ${ }_{3} k_{1}=0.2 \mathrm{~m} \mathrm{~d}^{-1}$ bar $^{-2}$; the fit was not improved by taking $q \neq 1$. The laboratory data for sliding over rough granite [Budd et al., 1979, Figure 7] give ${ }_{3} k_{1}$ in the range $0.4-1.1 \mathrm{~m} \mathrm{~d}^{-1} \mathrm{bar}^{-2}$; the nonconstancy of ${ }_{3} k_{1}$ can be corrected by taking $q=1.5$, with ${ }_{3} k_{1.5}=2.5 \mathrm{~m} \mathrm{~d}^{-1} \mathrm{bar}^{-1.5}$. From fitting (34) to ice sheet data, Budd et al. [1984] chose $p=1, q=2$, whereas Lingle and Brown [1986] chose (35) but took ${ }_{3} k_{1}$ to vary over a wide range (a factor of $\sim 100$ ), so that the choice $p=3, q=1$ is moot in this case. The sliding conditions at the base of Variegated Glacier in surge are probably rather different from those present in the laboratory experiments and the field situations cited; hence one does not necessarily expect a sliding relation in agreement with these other studies, which are moreover not fully consistent among themselves, as just noted. To represent by means of (34) the observed range of surge velocities $\sim 1-10 \mathrm{~m} / \mathrm{d}$ for water level depths in the range $100-70 \mathrm{~m}$ (exclusive of transitory peaks to higher levels) [Kamb et al., 1985, Figures 5 and 9] one finds that $q \approx 3$ is appropriate. Hence I will use

$$
v=v_{\mathrm{R}}\left(\frac{\tau}{\tau_{\mathrm{R}}} \cdot \frac{\sigma_{\mathrm{R}}}{\sigma}\right)^{3}
$$

with $\nu_{R}=1 \mathrm{~m} / \mathrm{d}$ and $\sigma_{R}=6$ bars (corresponding to a water level of $100 \mathrm{~m}$ depth in ice $400 \mathrm{~m}$ thick). The evaluation of the linked cavity model in this section is based on (36), with $\tau$ taken equal to $\tau_{R}$ because we consider here the hydraulic behavior in the surging state and choose the reference value $\tau_{R}$ to be the basal shear stress in the surging state; this restriction is relaxed in section 12 . An evaluation based on (35) gives overall results not greatly different from those given here on the basis of (36), although there are some interesting and illuminating differences in detail; these will be discussed in a subsequent paper.

When (36) and (7) and the parameter values stated above are introduced into (17) and (18), there results

$$
\Xi=\frac{2^{1 / 3}}{\pi^{1 / 2}} \frac{(\alpha \Lambda \omega)^{3 / 2}}{D M}\left(\frac{\eta_{\mathrm{R}}}{v_{\mathrm{R}} \sigma_{\mathrm{R}}}\right)^{\frac{1}{2}} h^{\frac{7}{6}}\left(\frac{\tau_{\mathrm{R}}}{\tau}\right)^{\frac{3}{2}}=0.18
$$

and

$$
\begin{gathered}
Q_{\mathrm{w}}=\frac{2^{4 / 3}}{\pi^{1 / 2}} \frac{N_{\mathrm{o}}}{M}\left(\frac{\alpha \Lambda}{\omega}\right)^{\frac{1}{2}}\left(\frac{\eta_{\mathrm{R}} \nu_{\mathrm{R}}}{\sigma_{\mathrm{R}}}\right)^{\frac{1}{2}} h^{\frac{13}{6}} \Phi\left(\frac{\tau}{\tau_{\mathrm{R}}}\right)^{\frac{3}{2}}\left(\frac{\sigma_{\mathrm{R}}}{\sigma}\right)^{3} \\
Q_{\mathrm{w}}=0.63\left(\frac{6}{\sigma}\right)^{3} \mathrm{~m}^{3} / \mathrm{s}
\end{gathered}
$$

where $\sigma$ is in bars; $\Phi$ in (38) is 0.47 , from Figure 7 , for the value of $\Xi$ found in (37). The dependences on $\sigma$ assumed for $\eta$ and $v$ in (7) and (36) lead to the result, in (37), that $\Xi$ does not vary with $\sigma$ and has the constant value 0.18 .

The $Q_{W}$-versus- $\sigma$ curve for the step-orifice system in Figure 12 is a plot of $(38 b)$. The orifice breadth $l_{R}$ at the reference condition $\sigma=\sigma_{R}$ is $l_{R}=1.4 \mathrm{~m}$, from (5). If we introduce (7) and (36) into (5) and recognize that $m$ is constant because $\Xi$ is constant, we obtain $l / l_{R}=\left(\sigma_{R} / \sigma\right)^{3}$, so that the gap length $l$ increases rapidly with the water pressure. At $\sigma=3$ bars it has lengthened to $l=10.8 \mathrm{~m}$. It is this lengthening that mainly causes the increase in $Q_{\mathrm{W}}$ with $P_{\mathrm{W}}$ given by (38) and shown by the step orifice curve in Figure 12.

Quantitative evaluation of the wave orifice linked cavity model, giving the corresponding curve of $Q_{\mathrm{w}}$ versus $\sigma$ in Figure 12, is carried out in a similar way. It is done with the parameters

$$
\begin{array}{ll}
\lambda=2 \mathrm{~m} & \text { bedrock surface wavelength } \\
a=7.8 \mathrm{~cm} & \text { sine wave amplitude } \\
\Sigma_{\mathrm{o}}=12 \text { bars } & \text { wave orifice cavitation limit (see below) }
\end{array}
$$

and with other parameters as listed above. The viscosity $\eta$ is given by (12); it does not appear explicitly, but it affects the value of the wave cavitation parameter $\Sigma$ given by (13). For the parameters stated, orifice cavitation starts at $\sigma=\Sigma_{0}=12$ bars. This is calculated by substituting $v$ from (36) into (13), putting $\sigma=\Sigma=\Sigma_{0}$, and solving for $\Sigma_{0}$, with the result

$$
\Sigma_{\mathrm{o}}=\left(2 N \sigma_{\mathrm{R}}\right)^{\frac{1}{2}}\left(\frac{2 \pi e}{\lambda}\right)^{\frac{1}{3}}\left(a v_{\mathrm{R}}\right)^{\frac{1}{6}}\left(\frac{\tau}{\tau_{\mathrm{R}}}\right)^{\frac{1}{2}}
$$

$\Sigma_{\mathrm{o}}$ is therefore not an independent parameter, being given in terms of the other parameters by (39). I call $\Sigma_{0}$ the wave cavitation limit, as distinct from the wave cavitation parameter $\Sigma$. From (13) and (36) it also follows that for any $\sigma$, 


$$
\Sigma=\Sigma_{\mathrm{o}}^{2} / \sigma
$$

and hence the ratio $\sigma / \Sigma$ that appears in (24)-(29) is

$$
\sigma / \Sigma=\left(\sigma / \Sigma_{0}\right)^{2}
$$

When (36) and (40b) are introduced into (25) and (29) and evaluated with the parameters given, we obtain

$$
\begin{gathered}
\Xi^{\prime}=0.162 \frac{(\alpha \Lambda / \omega)^{3 / 2}}{D M} \frac{\lambda a^{2 / 3}}{v_{\mathrm{R}}}\left(\frac{\tau_{\mathrm{R}}}{\tau} \frac{\sigma}{\sigma_{\mathrm{R}}}\right)^{3}\left(1-\frac{\sigma^{2}}{\Sigma_{\mathrm{o}}^{2}}\right)^{\frac{11}{6}} \\
\Xi^{\prime}=0.21\left(\frac{\sigma}{\sigma}\right)^{3}\left(1-\frac{\sigma^{2}}{12^{2}}\right)^{\frac{11}{6}}
\end{gathered}
$$

where $\sigma$ is in bars, and

$$
\begin{gathered}
Q_{\mathrm{W}}=1.38 \frac{N_{\mathrm{o}}}{M}\left(\frac{\alpha \Lambda}{\omega}\right)^{\frac{1}{2}} \lambda a^{\frac{5}{3}}\left(1-\frac{\sigma^{2}}{\Sigma_{\mathrm{o}}^{2}}\right)^{\frac{23}{6}} \Psi \\
Q_{\mathrm{w}}=9.8\left(1-\frac{\sigma^{2}}{12^{2}}\right)^{\frac{23}{6}} \Psi \quad \mathrm{m}^{3} / \mathrm{s}
\end{gathered}
$$

with $\sigma$ again in bars. The wave orifice curve in Figure 12 is a plot of $(42 b)$. The flux factor $\Psi$ is from Figure 9 on the basis of $\Xi^{\prime}$ values calculated from $(41 b)$. For the wave curve, unlike for the step, the melting-stability parameter $\Xi^{\prime}$ varies with $\sigma$, as (41) indicates, from 0 to a maximum of $\Xi^{\prime}=0.17$, at $\sigma=8$ bars.

The wave orifice gap length at $\sigma=6$ bars, as calculated from (26) with the parameters stated, is $l=1.43 \mathrm{~m}$. Two thirds of the gap lengthening takes place between $\sigma=12$ bars and 9 bars. At $\sigma=6$ bars the height of the gap at its widest point is $9.2 \mathrm{~cm}$, from (20) and Figure 10.

From (1) it is readily calculated that the Reynolds number for water flow in orifices of the linked cavity model evaluated above is greater than the turbulent flow threshold value of about $2 \times 10^{3}$ provided that the gap height is greater than $3 \mathrm{~mm}$. The step orifice and wave orifice gaps discussed above are mostly higher than this, so that it is appropriate to use (1) as the basis for calculating the water flow through them.

At a gap height of $5 \mathrm{~cm}$, typical of the orifices considered, the mean water flow velocity from (1) is $0.4 \mathrm{~m} / \mathrm{s}$. This is much larger than the observed mean water transport velocity in surge, $0.025 \mathrm{~m} / \mathrm{s}$, which reflects the fact, discussed in section 3 , that the conduit system must consist of large cavities linked by narrow orifices. As long as the cavity dimensions $l_{c}$ (breadth) and $g_{c}$ (height) and the number of cavities $N_{c}$ in any cross section of the glacier are large enough to provide the needed cross-sectional area of $200 \mathrm{~m}^{2}$ (section 3), that is, as long as $N_{\mathrm{c}} l_{c} g_{\mathrm{c}}=200 \mathrm{~m}^{2}$, the cavity geometry is not further constrained. The constraint seems readily satisfied: if $N_{\mathrm{c}}=N_{\mathrm{o}}=50$, the cavities could be $0.5-1 \mathrm{~m}$ high and 4-8 $\mathrm{m}$ broad, which seems reasonable for heavy cavitation over a rough bed at high water pressure and high sliding velocity.

For comparison with the linked cavity model, the hydraulic performance of an ice tunnel is shown in Figure 12 in terms of the steady state flux-versus-water-pressure curve, calculated from

$$
Q_{\mathrm{w}}=\frac{\pi}{2^{6}} D^{4} M^{3}\left(\frac{\omega}{\alpha}\right)^{\frac{11}{2}}\left(\frac{\sigma}{3 N}\right)^{12}
$$

with the same values of $\alpha, M$, and $N$ used above and with $\omega=3$, which is compatible with the results of dye tracing after surge termination $\left(Q_{\mathrm{w}}=40 \mathrm{~m}^{3} / \mathrm{s}, \bar{u}_{\mathrm{w}} / \omega=0.7 \mathrm{~m} / \mathrm{s}\right)$. Equation (43) is closely related to equation (11) of Röthlisberger [1972], differing only in that it is derived for the condition $\alpha_{\mathrm{w}}=\alpha$ (hydraulic grade line parallel to the glacier surface), rather than for a horizontal conduit. These equations are for an assumed circular cylindrical tunnel shape.

\section{INTERPRETATION: THE WATER FLUX/PRESSURE RELATION—COMPARISON OF LINKED CAVITY AND TUNNEL MODELS}

Whereas in a tunnel system the water flux at steady state is a decreasing (inverse) function of water pressure, in a linked cavity system it is an increasing (direct) function, as Figure 12 shows. This holds for linking orifices of either step cavity or wave cavity type, although the form of the function is rather different for the two. A linked cavity system containing orifices of both types has a flux-versus-pressure relation that is a combination of the two curves and again has $Q_{\mathrm{W}}$ increasing with $P_{\mathrm{W}}$. Qualitatively this result is independent of the details of the sliding-versus-pressure relation (36), as long as $v$ is an increasing function of $P_{\mathrm{W}}$.

An important consequence of the direct dependence of $Q_{\mathrm{w}}$ on $P_{W}$ is that a linked cavity system, unlike a tunnel system, can exist stably as a system of many interconnected conduits. In a system of interconnecting tunnels, the smaller tunnels are unstable with respect to the larger ones, which enlarge, capturing the drainage, while the smaller tunnels close up [Röthlisberger, 1972, p. 180; Shreve, 1972, p. 209]. The system tends to evolve to a single trunk tunnel. If the linked cavity system were subject to the same type of instability, the widespread network of interconnected cavities visualized in Figure 1 could not persist. By the same reasoning that shows that this type of instability is a feature of conduit systems in which the steady state flux-versus-pressure relation is inverse, it can be shown that the instability does not arise if the flux-versus-pressure relation is direct. This shows that a multiple conduit system of linked cavities, widely dispersed across the glacier bed and carrying the glacial water flux, can really exist and persist, contrary to what would be expected from the previously known properties of tunnel conduits.

A second important feature of the flux-versus-pressure relations in Figure 12 is that for a water flux greater than $\sim 1 \mathrm{~m}^{3 / \mathrm{s}}$ the linked cavity model requires much higher basal water pressures than the tunnel model does. This provides an explanation for why the basal water pressure is high in the surging state of glacier motion: a tunnel system is absent, and the linked cavity system that is present requires high water pressures in order to open up the orifice cavitation sufficiently to carry the basal water flux furnished by water input upstream. The distinction in Figure 12 between water level depths of $\sim 60-90 \mathrm{~m}$ in the linked cavity model and $>180 \mathrm{~m}$ in the tunnel model, for water fluxes in the range $2-20 \mathrm{~m}^{3 / \mathrm{s}}$, parallels the distinction between observed water levels in the surging and nonsurging states [Kamb et al., 1985, Figure 9]. (In fact, the water levels calculated for the tunnel system are somewhat lower than the average of those actually observed after surge termination, corresponding better with the lowest levels observed. A somewhat similar situation was noted by Iken and Bindschadler [1986, pp. 113-114] in calculated water levels for basal tunnels in Findelen Glacier, Switzerland. A lower value of $N$ in (43), such as the value 0.50 bar $\mathrm{yr}^{1 / 3}$ chosen by Röthlisberger [1972, p. 194] or the value 0.81 bar $\mathrm{yr}^{\mathrm{t} / 3}$ advocated by Lliboutry [1983, p. 220], gives water levels nearer to the mean level (depth 110-130 m) in Variegated Glacier after surge.) 
The conclusion that basal water pressures are much higher in a linked cavity system than in a tunnel system at water flux levels $\geq 1 \mathrm{~m}^{3 / \mathrm{s}}$ holds for step heights $h$ and wave amplitudes $a$ of order $10 \mathrm{~cm}$ or less, such as those used in the model evaluations in section 9. Because $Q_{\mathrm{W}}$ is an increasing function of $h$, or of $a$ and $\lambda$, as shown by (18) and (29) or by (38) and (42), with large enough values of these dimensions it is possible to increase $Q_{\mathrm{W}}$ at fixed $\sigma$ by arbitrarily large amounts and therefore to decrease correspondingly the water pressure at a given flux to values as low as or lower than for a tunnel with the same flux. For example, by increasing $h$ in the step model from 5 to $22 \mathrm{~cm}$, the water level at $Q_{W}=10 \mathrm{~m}^{3 / \mathrm{s}}$ is lowered from 64 to $190 \mathrm{~m}$. Changing other parameters in (38) and (42) could also contribute to lowering the water pressure. It follows that the surging state of glacier motion, made possible by the presence of a linked cavity basal water conduit system at high water pressure, is achievable only on beds of certain roughness characteristics, that is, certain dimensions and spatial arrangements of roughness features, equivalent statistically to what is achieved in the step orifice model in idealized form by arranging steps of height $\sim 5 \mathrm{~cm}$ among larger roughness forms in such a way as to provide orifices linking the leeside cavities behind the larger features.

The presence of the large cavities in the basal water conduit system is not a feature necessary for the foregoing conclusion: putting $\Lambda$ equal to 1 in (38a) and (42a), which would mean no large cavities, decreases $Q_{\mathrm{w}}$ only by a factor of about 3 , which could be compensated by a change in the dimensional parameters or $N_{0}$. The necessity of the cavities in the linked cavity system comes not from their effect on the $Q_{\mathrm{w}}$-versus- $P_{\mathrm{w}}$ curves but rather from the observational evidence on dye transport through the system and also from the logical expectation that the cavitation gap heights and breadths over an actual bed surface will be highly variable from place to place, creating naturally a system of large cavities interspersed with narrow orifices (section 3).

A linked cavity conduit system can form over a "soft" bed consisting largely of unconsolidated rock debris provided the bed has a sufficient number of roughness features that do not move with the ice, such as bedrock protuberances or large protruding boulders that are stuck at deeper levels so that they do not move with deformation of the "soft" parts of the bed. The local conditions for cavitation, and the shape of the cavities and orifices that form, are not fundamentally different from those over a "hard" (bedrock) bed. Consequently, one can expect that a linked cavity system on a soft bed at high speeds of ice motion will have a flux-versus-pressure relation that is similar, at least qualitatively, to the curves for the hard beds modeled above and also that it will be subject to a melting-instability parameter similar to $\Xi$ or $\Xi^{\prime}$ in section 8. Such a conduit system must be present whether or not soft bed deformation is involved in the surge motion because the water flux in spring or summer is much too large to be transmitted by a layer of subglacial till or any granular medium except very coarse gravel [Iken and Bindschadler, 1986, p. 104; Shoemaker, 1986]. It follows that the surge mechanism modeled here on the basis of hard beds is also applicable in a general way to surging when soft bed deformation contributes to the high speeds of ice motion.

\section{STABILITY/INSTABILITY FEATURES OF LINKED CAVITY AND TUNNEL SYSTEMS}

The stability or instability of a multiple conduit system and its relation to direct or inverse flux-versus-pressure functions, discussed in section 10 , is a secondary manifestation of what I will call primary stability/instability of conduits. A tunnel at steady state under fixed hydraulic gradient is unstable against perturbations in size or water pressure: if the size, or the water pressure, is increased, the tunnel will grow, at an ever accelerating rate, because the wall melting rate increases faster than the tunnel closure rate as the size increases; conversely, if the size or the pressure is reduced, the tunnel will contract and will close up completely, for the same reason with change of sign. (The growth instability manifests itself in the jökulhlaup phenomenon [Nye, 1976; Spring and Hutter, 1981; Clarke et al., 1984].) In contrast, the orifices of a linked cavity system in steady state under fixed hydraulic gradient are stable against infinitesimal perturbations of this type according to the model developed here: if the size is perturbed, the response is a retum toward the original steady state size, and if the pressure is perturbed, the response is a measured adjustment toward a new steady state size dependent on the new pressure.

The feature that provides the basis for the primary stability of the linked cavity system is the flow capacity of the system in the absence of melting by viscous dissipation, when the conduits are formed entirely by cavitation under the available water pressure. No corresponding feature is present in the model concept of a tunnel system [Röthlisberger, 1972]. One of the reasons for developing the model of the linked cavity system in sections 4-8 is to find out whether or not the stabilizing feature provided by cavitation persists when viscous heat dissipation is introduced into the linked cavity system. The results in section 8 show that it does, within limits.

The limits are set by the melting-stability parameter $\Xi$ (for step orifices) or $\Xi^{\prime}$ (for wave orifices), as discussed in section 8 . These parameters provide a measure of the magnitude of the viscous dissipation, as shown by the direct connection between them and the amount of orifice roof meltback $m$, tied to parameters $\mu$ and $v$ in Figures 7 and 9. The stabilizing effect of the basal cavitation phenomenon predominates as long as the magnitude of the viscous heating, measured by $\Xi$ or $\Xi^{\prime}$, is small enough, that is, as long as $\Xi \leq 1$ and $\Xi^{\prime} \leq 1.5$. The instability that develops in a step orifice for $\Xi_{z} 1$ or in a wave orifice for $\Xi^{\prime} \geq 1.5$ manifests itself when viscous heating results in an accelerating, uncontrolled orifice growth that is essentially the same (and with the same cause) as the accelerating growth of a tunnel, described above. However, this growth instability of orifices differs from that of tunnels in that orifices are unstable only under finite perturbations from the steady state. The required size of the destabilizing perturbation decreases with increase of $\Xi$ or $\Xi^{\prime}$, and becomes small for $\Xi \approx 1$ and $\Xi^{\prime} \approx 1.5$, as seen for the step orifice in Figure 11 . For tunnels there is no stability parameter analogous to $\Xi$ or $\Xi^{\prime}$; tunnels are unstable against even infinitesimal perturbations under all conditions of fixed hydraulic gradient. This is because there exists no steady state for tunnels of finite diameter in the absence of viscous heating. Finally, whereas the tunnel instability is bilateral (i.e., it applies for either positive or negative perturbations), the orifice instability occurs only for an increase in size or water pressure from the steady state condition. We see here a fundamental distinction between the hydraulic behavior of the orifices of a linked cavity system and the tunnels of a normal tunnel system.

Figure 13 shows a visualization of what happens when a step orifice goes into unstable growth. It evolves into a tunnel, which is carried downstream with the movement of the ice. The upstream part of the enlarging tunnel begins to collapse and closes off, leaving a step orifice behind, while the tunnel is carried away. What happens to it thereafter is a complicated matter, dependent on the three-dimensional complexities of the bed geometry. Without entering into the numerous possibilities and complica- 


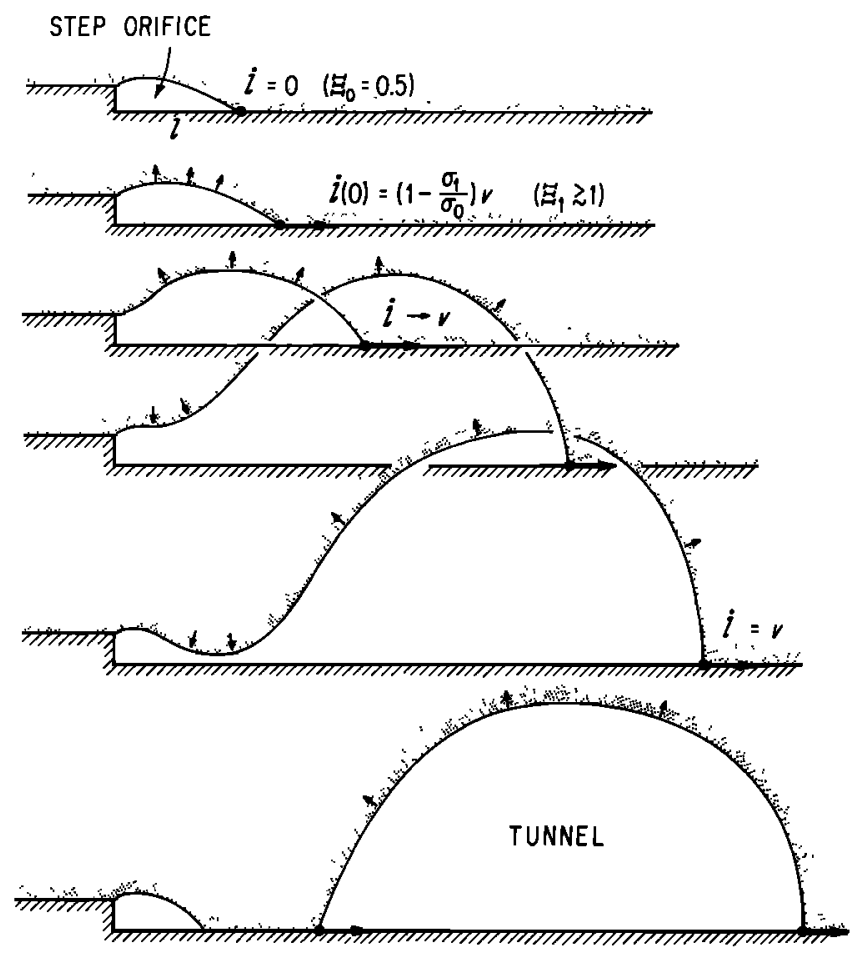

Fig. 13. Schematic picture of what happens when a step orifice goes unstable for $\Xi \geq 1$, shown in a time sequence of diagrams with time increasing from top to bottom. The bed is marked with cross hatching and the ice is shown stippled, in the style of Figures 4 and 5 . At the top the step orifice is shown in steady state under some effective confining pressure $\sigma_{0}$, and with $\Xi$ approximately 0.5 ; the recontact point is not moving, that is, $l=0$, where $l$ is the orifice gap length. Upon increase of $\Xi$ to a value greater than about 1 , and upon decrease in effective pressure to $\sigma_{1}<\sigma_{0}$ as in Figure 11, the orifice starts to expand as shown by the arrows in the second diagram; initially the gap length increases at a rate $i(0)=\left(1-\sigma_{1} / \sigma_{0}\right) \nu$, where $v$ is the sliding velocity (see section 8 ). As the expansion continues, the gap lengthening rate approaches the maximum possible value $l \rightarrow v$ as noted in the third diagram. When the gap length passes the steady state length $l_{1}$ that corresponds to $\sigma_{1}$ in accordance with Figure 7 for the perturbed parameter $\Xi_{1}$, the head of the cavity begins to collapse, as indicated in the fourth snapshot, but the enlarged orifice downstream continues to grow rapidly by melting under greatly increased water flow and becomes effectively a tunnel segment connecting the two cavities that were initially connected by the step orifice. Ultimately the head of the cavity closes off, as shown in the last snapshot, and the tunnel segment is advected downstream at speed $v$. If it does not encounter a large stoss slope downstream and get pinched off and if it can swing around into a longitudinal orientation, it can become a more or less permanently established tunnel segment in the basal water conduit system, provided that the local water flux and local water pressure adjust to the levels needed for its stable maintenance as a tunnel in accordance with (43).

tions, I surmise that if viscous heating in the system is large enough ( $\Xi$ or $\Xi^{\prime}$ large enough) and if the initiating perturbation is big enough that a large enough number of orifices go into unstable growth simultaneously, a network of interconnected tunnels may start to develop out of cavities linked by unstably growing orifices or by the tunnel segments shed downstream by them. If this development is able to continue, which probably requires that a large enough part of the bed be affected to allow the process to develop collectively and cooperatively, the linked cavity system can thereby convert to a multiple-tunnel system, which then proceeds to degenerate into a single-tunnel system by multiple-conduit instability. In my view, this is the process of surge termination, by which the basal water conduit system converts from a linked cavity system to a tunnel system, with consequent drop in basal water pressure and slowdown of the sliding speed. It is noteworthy that the observed surge termination was immediately preceded by a high peak in water pressure [Kamb et al., 1985, Figure 9], which could have provided the initiating perturbation that put the orifices into unstable growth.

The values of the melting-instability parameters derived for the linked cavity models in section $9, \Xi=0.18$ for the step orifice model and $\Xi^{\prime} \leq 0.17$ for the wave orifice model, from (37) and (41), are enough smaller than 1 to assure that these model linked cavity systems could exist stably at the glacier bed. At the same time $\Xi$ and $\Xi$ ' are within "shooting distance" of 1 , so that for reasonable changes in the model input parameters in section 9 it is possible to reach a condition where $\Xi>1$ or $\Xi^{\prime}>1.5$, for which the linked cavity systems would be unstable and would convert to tunnel systems, as discussed above.

\section{SURGE MECHANISM}

The above considerations imply that it is possible for some glaciers to be in a state of rapid sliding motion with a linked cavity basal water conduit system at high basal water pressure, while for others the development of a tunnel system with low water pressure precludes such a state. Moreover, it is possible for a glacier to make a transition to this state and back again. This is the essence of a surge mechanism.

The possibility of a surging state of glacier motion is predicated upon two features of the linked cavity system: (1) the high basal water pressure (and consequent high basal sliding velocity) that the linked cavity configuration can force upon the basal water system at typical water transport fluxes of order $1-10 \mathrm{~m}^{3 / \mathrm{s}}$ or larger, as discussed in section 10 , and (2) the possible stability of the multiple conduit linked cavity configuration against conversion to a single-conduit tunnel system through the action of viscous heat dissipation, discussed in sections 10 and 11. For this type of surging state to be possible for a given glacier, it is necessary that the water-flux-versus-water-pressure curve for its basal cavitation system be such as to require appropriately high basal water pressures ( $2-5$ bars below ice overburden pressure in the case of Variegated Glacier) at the current water flux levels imposed by input of water to the basal water system from all sources, and it is necessary that the melting-stability parameter ( $\Xi$ or $\Xi^{\prime}$ in sections 7 and 8 ) for its linked cavity system under these current conditions be less than a certain critical value that separates stability (low $\Xi$ ) from instability (high $\Xi$ ) against conversion to a tunnel system. These conditions can be satisfied only if the system parameters lie within certain ranges, and this is what, in principle, distinguishes glaciers that can surge from ones that cannot surge. The linked cavity model in sections 7-11 provides a means of identifying in simplified form what the relevant system parameters are, and of showing that for reasonable values of these parameters (such as those listed in section 9) the conditions for the surging state can be realized, while for other reasonable values the conditions are not satisfied (sections 10 and 11). Since the "orifices" (section 3) of the linked cavity system control the water flow, their dimensional parameters are particularly important; in general, the roughness features that generate the orifices by basal cavitation must have small amplitude $(\leqslant 0.1 \mathrm{~m})$ in order that the conditions for surging can be satisfied. Also important is the sliding velocity, because rapid sliding stabilizes the orifices, by decreasing $\Xi$ in (17) and $\Xi^{\prime}$ in (25). Sliding of $\sim 1 \mathrm{~m} / \mathrm{d}$ under a 
basal water pressure about 6 bars below overburden is about sufficient to confer stability upon orifices generated by roughness features of amplitude $\sim 0.1 \mathrm{~m}$, but if the sliding velocity were a fifth this great, the orifices would be unstable, according to the model results in section 9 . Since the sliding velocity at any given basal shear stress and water pressure will increase as the bed roughness is decreased, low roughness again favors surging, but the roughness that is relevant here is probably not that associated with the orifices but rather the larger-amplitude roughness associated with wave cavities of the linked cavity system.

Although the distinction between "surgeable" and "nonsurgeable" glaciers thus seems in principle clear, there is a large gap between these principles and their practical application to the problem of explaining why certain glaciers surge and others do not. For one thing, the relationship between the simple model parameters and the complex actual features of real glacier beds is problematical, and also there is in general no way to measure such parameters at the bottom of actual glaciers. For another thing, the connection between the system parameters and the basal sliding relation ((36b) or (36c) or other) is a complicated matter. This latter problem can be dealt with to some extent for examples like Variegated Glacier and Findelen Glacier for which there exist observational data for an empirical relation between water pressure and sliding up to the high values relevant for surging. For glaciers that do not experience high basal water pressures, the needed empirical relation would be unobtainable.

Despite these difficulties, a test of the ability of the linked cavity model to distinguish predictively in a statistical way between surge-type and nonsurge-type glaciers is provided by the observed correlation between glacier length and probability of surging in a large sample of Yukon glaciers [Clarke et al., 1986, Figure $2 b$ ]. Glacier length does not directly enter as a parameter in the model, but since there is a strong inverse correlation between length and surface slope in the sample studied [Clarke et al., 1986, Figure 4a], the observed correlation can be considered an inverse correlation between surge probability and slope $\alpha$, which is a parameter in the model. (Clarke et al. [1986] concluded that the correlation was really with length alone and that slope had no effect, but a compelling statistical argument that would require this conclusion was not given.) The parameters $\Xi$ in (37) and $\Xi^{\prime}$ in (41a) vary as $\alpha^{3 / 2}$; hence other things being equal, the model indicates lower values of $\Xi$ and $\Xi^{\prime}$ for longer glaciers, which means a higher probability of values low enough to stabilize a linked cavity system and allow surging. Thus qualitatively, at least, the model passes this test.

The question of how and why a surge-type glacier makes the transition from the nonsurging state to the surging state and back again is central to the mechanism of surging. It is suggested in section 11 that surge termination occurs when the linked cavity system goes unstable and converts to a tunnel system. The simplest mechanism of surge initiation, within the framework provided by the linked cavity model, is that the basal water conduit system first becomes a linked cavity system without tunnels, which happens in winter, when the water flux is low enough, and then, when the flux increases in spring, $\Xi$ happens to be low enough that the linked cavity system does not degenerate to a tunnel system. The lowering of $\Xi$ to the point where the linked cavity system is stabilized can be caused in the reservoir area of the glacier by the increase in basal shear stress there as the ice thickness builds up prior to surge: (37) and (41a) show that an increase in $\tau$ decreases $\Xi$ and $\Xi^{\prime}$. In agreement with this concept for surge initiation, the surge of Variegated Glacier started in the reservoir area. The effect of increased $\tau$ would to some extent be offset by an increase of $\alpha$ in (37) and (41a), but in fact $\alpha$ did not increase in the area where the surge actually started (C. F. Raymond, personal communication, 1986; C. F. Raymond et al., Variegated Glacier studies-1979, unpublished manuscript, Figure 3).

The proposed surge mechanism, as described above, involves entry into the surging state in winter. This is in agreement with the fact that the surge of Variegated Glacier did start in wintertime. However, the attainment of a surge speed of $2 \mathrm{~m} / \mathrm{d}$ or more in midwinter, when the availability of water for the basal water system is minimal, indicates that there is more to the surge initiation mechanism than the foregoing discussion suggests. The concepts of the linked cavity model prove useful in considering in greater detail the cause of wintertime surge initiation, which will be taken up in a subsequent paper.

Acknowledgments. This work was supported by grants DPP-8209824 and DPP-8519083 from the U.S. National Science Foundation. I thank Paul Hawley for the text processing. Caltech Division of Geological and Planetary Sciences contribution 4428.

\section{REFERENCES}

Bindschadler, R., The importance of pressurized subglacial water in separation and sliding at the glacier bed, J. Glaciol., 29, 3-19, 1983.

Brugman, M. M., Water flow at the base of a surging glacier, Ph.D. thesis, Calif. Inst. of Technol., Pasadena, 1986.

Budd, W. F., P. L. Keage and N. A. Blundy, Empirical studies of ice sliding, J. Glaciol., 23, 157-170, 1979.

Budd, W. F., D. Jensen and I. N. Smith, A three-dimensional time-dependent model of the West Antarctic ice sheet, Ann. Glaciol., 5, 29-36, 1984.

Carol, H., The formation of roches moutonnées, J. Glaciol., 1, 57-59, 1947.

Clarke, G. K. C., W. H. Mathews and R. T. Pack, Outburst floods from glacial Lake Missoula, Quat. Res., 22, 289-299, 1984.

Clarke, G. K. C., J. P. Schmok, S. L. Ommaney and S. G. Collins, Characteristics of surge-type glaciers, J. Geophys. Res., 91, 7165-7180, 1986.

Engelhardt, H. F., W. D. Harrison and B. Kamb, Basal sliding and conditions at the glacier bed as revealed by borehole photography, J. Glaciol., 20, 469-508, 1978.

Fowler, A. C., A sliding law for glaciers of constant viscosity in the presence of subglacial cavitation, Proc. R. Soc. London, Ser. A, 407, 147-170, 1986.

Hallet, B., and R. S. Anderson, Detailed glacial geomorphology of a proglacial bedrock area at Castleguard Glacier, Alberta, Canada, Z. Gletscherk. Glazialgeol., 16, 171-184, 1980.

Iken, A., The effect of subglacial water pressure on the sliding velocity of a glacier in an idealized numerical model, $J$. Glaciol., 27, 407-421, 1981.

Iken, A., and R. A. Bindschadler, Combined measurements of subglacial water pressure and surface velocity of Findelengletscher, Switzerland: Conclusions about drainage system and sliding mechanism, J. Glaciol., 32, 101-119, 1986.

Kamb, B., Sliding motion of glaciers: Theory and observation, Rev. Geophys., 8, 673-728, 1970.

Kamb, B., and H. Engelhardt, Waves of accelerated motion in a glacier approaching surge: The mini-surges of Variegated Glacier, Alaska, J. Glaciol., 33, 27-46, 1987.

Kamb, B., and E. R. La Chapelle, Direct observation of the mechanism of glacier sliding over bedrock, J. Glaciol., 5 , 159-172, 1964. 
Kamb, B., C. F. Raymond, W. D. Harrison, H. Engelhardt, K. A. Echelmeyer, N. Humphrey, M. M. Brugman and T. Pfeffer, Glacier surge mechanism: 1982-1983 surge of Variegated Glacier, Alaska, Science, 227, 469-479, 1985.

Lingle, C. S., and T. J. Brown, A subglacial aquifer bed model and water-pressure-dependent basal-sliding relationship for a west-Antarctic ice stream, in The Dynamics of the West Antarctic Ice Sheet (ed. C. J. van der Veen and J. Oerlemans), pp. 249-285, D. Reidel, Dordrecht, 1986.

Lliboutry, L., General theory of subglacial cavitation and sliding of temperate glaciers, J. Glaciol., 7, 21-58, 1968.

Lliboutry, L., Modification to the theory of intraglacial waterways for the case of subglacial ones, J. Glaciol., 29, 216-226, 1983.

Nye, J. F., The flow law of ice from measurements in glacier tunnels, laboratory experiments and the Jungfraufirn borehole experiment, Proc. R. Soc. London, Ser. A, 219, 477-489, 1953.

Nye, J. F., A calculation of the sliding of ice over a wavy surface using a Newtonian viscous approximation, Proc. $R$. Soc. London, Ser. A, 311, 445-467, 1969.

Nye, J. F., Water at the bed of a glacier, IAHS Publ., 95, 189-194, 1973.

Nye, J. F., Water flow in glaciers: jökulhlaups, tunnels, and veins, J. Glaciol., 17, 181-207, 1976.

Paterson, W. S. B., The Physics of Glaciers, Pergamon, New York, 1981.

Raymond, C. F., How do glaciers surge? A review, J. Geophys. Res., this issue.

Röthlisberger, H., Water pressure in intra- and subglacial channels, J. Glaciol., 11, 177-203, 1972.
Shoemaker, E. M., Subglacial hydrology for an ice sheet resting on a deformable aquifer, J. Glaciol., 32, 20-30, 1986.

Shreve, R. L., Movement of water in glaciers, J. Glaciol., 11, 205-214 1972.

Spring, U., and K. Hutter, Numerical studies of jökulhlaups, Cold Reg. Sci. Technol., 4, 227-244, 1981.

Spring, U., and K. Hutter, Conduit flow of a fluid through its solid phase and its application to intraglacial channel flow, Int. J. Eng. Sci., 20, 327-363, 1982.

Vivian, R., The nature of the ice-rock interface: The results of investigation on $20,000 \mathrm{~m}^{2}$ of the rock bed of temperate glaciers, J. Glaciol., 25, 267-277, 1980.

Vivian, R., and G. Bocquet, Subglacial cavitation phenomena under the Glacier d'Argentière, Mont Blanc, France, J. Glaciol., $12,439-451,1973$.

Walder, J., and B. Hallet, Geometry of former subglacial water channels and cavities, J. Glaciol., 23, 335-346, 1979.

Weertman, J., On the sliding of glaciers, J. Glaciol., 3, 33-38, 1957.

Weertman, J., General theory of water flow at the base of a glacier or ice sheet, Rev. Geophys., 10, 287-333, 1972.

B. Kamb, Division of Geological and Planetary Sciences, Califomia Institute of Technology, Pasadena, CA 91125.

(Received November 17, 1986;

revised March 31, 1987;

accepted May 7, 1987.) 